\title{
Filter Design for Positive T-S Fuzzy Continuous-Time Systems with Time Delay Using Piecewise-Linear Membership Functions
}

\author{
Aiwen Meng, Hak-Keung Lam, Fellow, IEEE, Fucai Liu, Yingjie Yang
}

\begin{abstract}
This work focuses on the filtering problem and stability analysis for positive Takagi-Sugeno (T-S) fuzzy systems with time delay under $L_{1}$-induced performance. Due to the importance of estimation of system states but the few filter design results on positive nonlinear systems, it is an attractive and meaningful topic well worth studying. In order to fully exploit and take advantage of the positivity of positive T-S fuzzy systems, many commonly used methods, for instance free-weighting matrix approach and similarity transformation are probably not suitable for positive systems. To address the hard-nut-to-crack problem, an auxiliary variable is introduced so that the augmentation approach can be employed to carry out the positivity and stability analysis of filtering error systems. In addition, another obstacle that cannot be ignored is the existence of non-convex terms in the stability and positivity conditions. For getting around this barrier, some iterative linear matrix inequality (ILMI) algorithms have been proposed in the literature. However, considering the weakness that these methods cannot guarantee the convergence to a numerical solution and the iterative process is exhaustive, we present an effective matrix decoupling method to convert the nonconvex conditions into convex ones in this paper. Furthermore, a linear co-positive Lyapunov function which incorporates the positivity of system states and time delay at the same time is chosen so that the positivity characteristic of filtering error systems can be captured further. However, because of plenty of valuable information of membership functions (MFs) being ignored, hence, the obtained results are conservative. For the sake of relaxing the conservativeness, the advanced piecewise-linear membership functions (PLMFs) approximate method is utilized to facilitate the stability and positivity analysis. Therefore, the relaxed stability and positivity conditions which are cast as sum of squares (SOS) are obtained and can be solved numerically. Finally, the effectiveness of the designed fuzzy filtering strategy with satisfying $L_{1}$-induced performance are demonstrated by a simulation example.
\end{abstract}

Index Terms-Positive T-S fuzzy systems, filter design, $L_{1}$ induced performance, sum of squares (SOS).

\section{INTRODUCTION}

$\mathbf{I}$ $\mathrm{N}$ the last decade or so, researchers paid more attention to positive systems which have some special properties. For example, the state variables and system outputs will stay in

This work was supported in part by Natural Science Foundation of Hebei Province under Project Number F2019203505, China Scholarship Council, King's College London and De Montfort University.

Aiwen Meng and Fucai Liu are with the Key Laboratory of Industrial Computer Control Engineering of Hebei Province, Yanshan University, Qinhuangdao, Hebei 066004, China. (e-mail: 1309494495@qq.com; 1fc@ysu.edu.cn).

H. K. Lam is with the Department of Engineering, King's College London, Strand London WC2R 2LS, U.K. (e-mail: hak-keung.lam@kcl.ac.uk).

Yingjie Yang is with the Institute of Artificial Intelligence, Grateway House, De Montfort University, Leicester LE1 9BH, U.K. (e-mail: yyang@dmu.ac.uk). the positive quadrant if the initial conditions are non-negative [1]-[3]. Such systems are very close to our daily life, such as, in biological and physiological field: metabolic systems; in ecology and population dynamics: the human, animal and plant populations; in communications: data packets flowing in a network; and in compartmental systems: pharmacokinetics, epidemiology and so on. Therefore, it is very important and valuable to have a deep understanding for positive systems.

Different from general systems, positive systems are defined on cones rather than linear spaces. This kind of unique and elegant positivity will make numerous well-established results for general systems not applicable to positive systems. Therefore, an increasing number of researchers have focused on positive systems from different perspectives, such as the reachability and controllability [4]-[6], the realization [7], [8], the control synthesis, stability analysis and optimal control for positive systems [9]-[12]. Furthermore, different control strategies have been employed for positive systems, for instance, state feedback control strategy [13], [14], static output feedback control strategy [15], [16], observer design [17], positive filter design [18], [19] and so on.

The filter design for general systems has been attracting researchers for decades and rich results have been achieved [20]-[23]. In view of the various filtering results in the literature, we notice that the $L_{1}$ filtering and $H_{\infty}$ filtering [24], [25] are more popular than Kalman filtering [26], [27] because the formers can achieve a great filtering performance without the exact knowledge of the statistics of the external noise signals [28]. Another advantage of $L_{1}$ filtering and $H_{\infty}$ filtering is insensitivity to the uncertainties either in the exogenous signal statistics or in dynamic models. All of these benefits of the $L_{1}$ filtering and $H_{\infty}$ filtering make them more applicable to some practical systems. However, when designing filters for positive systems, people are more concerned about the total number of the components or the maximal quantities which can be captured by $L_{1}$ filtering rather than $H_{\infty}$ filtering. Thereby, from this point, the advantages of $L_{1}$ filtering are more obvious than $H_{\infty}$ filtering. To the best of our knowledge, although the $L_{1}$ filtering is helpful to describe the features of positive systems, the research results are not fruitful enough at present. Hence, it is one of the motivations for us to carry out this work.

On the other hand, although there are some results relating to filter design for positive systems, it is noted in the literature that most of the works are for positive linear systems [18], [19], [29]. In [18], positive filter for continuous-time positive systems under $L_{1}$ performance was investigated, meanwhile, 
a new feature of filter was discovered firstly to guarantee the stability and $L_{1}$-induced performance of the filter error system at the same time. The work in [19] mainly designed the event-triggered network-based $\ell_{1}$-gain filter for positive linear systems, in which the sufficient conditions were derived so that the positive linear filter existed with satisfying $\ell_{1}$ gain performance. In order to estimate the output of positive switched systems, the authors in [29] designed a positive filter based on both the upper-bound and lower-bound information of system states. Regrettably, as a matter of fact that many positive systems are of nonlinear in practical applications, such as the metabolic pathways [30], buck converter [31] and the gas-lifted oil well system [32], which means that the existing results for positive linear systems may be unsuitable for positive nonlinear systems [19], [33]. Therefore, the investigation of stability analysis and control design for positive nonlinear systems is well motivated and the achievements will expand the knowledge in the field.

Up to now, because of the complexity, high non-linearity and unique positivity constrain of positive nonlinear systems, the relevant results are less fruitful. Considering the case that only parts of the system states are available, we have investigated the static output feedback control synthesis for positive nonlinear based on fuzzy model in [34]-[36], but when all of the system states are not avaliable, these results using output feedback control strategy cannot be applied. Meanwhile, in consideration of the influence of external disturbance for system stability, designing positive fuzzy filters for positive nonlinear systems is of great significant and meaningful.

Fuzzy control belongs to one of the intelligent control methods and has been widely applied in the past several decades [37]-[40]. Especially, the popular Takagi-Sugeno (T-S) fuzzy model [41]-[45] has obvious superiorities in approximating complicated nonlinear systems. Nevertheless, the results related to the positive T-S filter design for positive nonlinear continuous-time systems using T-S fuzzy model are relatively few. From the literature, we find the work in [46] is mainly related to positive filter design for positive T-S fuzzy systems under $L_{1}$ performance, but it aims at the discrete-time systems without time delay. While the work in this paper mainly aims at positive T-S continuous-time systems with time delay. And from the analysis technique perspective, an auxiliary variable which is helpful to construct an augmented system will be introduced to facilitate the positivity and stability study, which is totally different from the analysis in [46]. Besides, it should be pointed out that time delay which not only can result in degradation of system performance but also is able to cause instability is frequently encountered in practical systems [47], such as, chemical reaction process, network transmission and reproduction of plants and animals. Hence, it is vital to take the time delay into account when the positive filter is designed for positive T-S continuous-time systems, which makes the results have a great of theoretical value and practical significance.

As mentioned before, we know that positive systems have the elegant property that all of the state variables are nonnegative values if the initial conditions are non-negative. Based on extensive literature review, it is shown that the linear copositive Lyapunov function not only can better capture the unique positivity of positive systems but also can reduce the difficulty of the analysis and computational burden [48]-[50]. In order to take advantage of this property, a linear co-positive Lyapunov function rather than a quadratic Lyapunov function is chosen to analyze the stability and positivity of the positive T-S fuzzy filter error systems in this paper. Up to now, as far as we know, there has been no $L_{1}$-induced positive filter design and stability analysis reported for positive T-S continuous-time systems with time delay by using linear co-positive Lyapunov function, which motivates us to study the topic to fill this gap.

At present, some advanced membership-function-dependent (MFD) techniques, for instance, staircase membership functions (MFs) [51], polynomial MFs [34], [52] and piecewiselinear MFs (PLMFs) [53], have been proposed to facilitate relaxed stability analysis. These techniques provide great help to extract the information of MFs, such as the shape information and boundary information. However, they usually are not taken into the stability analysis of positive T-S fuzzy systems because the MFs will lead to the complexity of stability analysis and MFD analysis is still in its early research stage [54]. In addition, when this advanced technique is employed for general fuzzy systems, the MFs $w_{i}(\mathbf{x})$ and $m_{j}(\mathbf{x})$ are only related to $\mathbf{x}$. However, in this paper, the MFs $w_{i}(\mathbf{x})$ and $m_{j}\left(\mathbf{x}_{f}\right)$ are related to $\mathbf{x}$ and $\mathbf{x}_{f}$, respectively, which means the complexity is greatly increased. Therefore, it is a very challenging task for us. On the other hand, trying to find a suitable approach to introduce the information of MFs into the stability analysis for positive T-S fuzzy filter systems incredibly stimulates us to perform the work.

In order to accomplish our purpose, there are some obstacles that require to be overcome: firstly, in order to capture the positivity of positive systems, it is more popular to analyze the stability by using the linear co-positive Lyapunov function compared with the quadratic Lyapunov function, but when using this advanced method, many useful techniques which are appropriate for quadratic Lyapunov function, such as the free-matrix approach and the similarity transformation might not appropriate for linear co-positive Lyapunov function. Therefore, in order to facilitate the positivity and stability analysis without the help of these methods, we will introduce an auxiliary variable which is in favor of the use of the augmentation approach [55]. Secondly, there are some nonconvex terms in the stability conditions, which make it hard to obtain the numerical solution based on the current state of the art. To break through this barrier, some iterative linear matrix inequality (ILMI) algorithms have been proposed. But it is well known that these methods cannot guarantee the convergence to a numerical solution and the iterative process is exhaustive. Thereby, we will develop a matrix decoupling method [18] to convert the non-convex conditions into convex ones so that this problem can be dealt with skillfully. Thirdly, for better capturing the positivity of the positive systems, $L_{1}$ induced performance index instead of $H_{\infty}$ performance is considered and a linear co-positive Lyapunov function candidate instead of a quadratic Lyapunov function candidate is chosen in this paper. Finally, some important information embedded in MFs is extracted and introduced into the stability analysis by employing the advanced PLMFs approximate technique so 
that the obtained stability and positivity conditions are relaxed.

The following is the arrangement of this paper. In Section II, we give a number of useful preliminaries and the positive filter design procedure. In Section III, the positivity and stability analysis is carried out for positive T-S fuzzy filter error systems. In Section IV, we show an example to verify the reliability and validity of the theoretical results. The Section $\mathrm{V}$ mainly shows the conclusion.

\section{PRELIMINARIES}

In this section, we will show some standard notations and give the positive T-S fuzzy model with time delay and the positive T-S fuzzy filter mathematically.

\section{A. Notation}

Over the course of the entire paper, the following notations are employed. For a matrix $\mathbf{M} \in \Re^{l \times n}$ where $m_{r s}$ denotes the element on $r$-th row and $s$-th column. $\mathbf{M} \succeq 0, \mathbf{M} \succ$ $0, \mathbf{M} \preceq 0$ and $\mathbf{M} \prec 0$ represent that each element $m_{r s}$ is non-negative, positive, non-positive and negative, respectively. $\mathbf{Q}(\mathbf{x})=\operatorname{diag}\left(x_{1}, \ldots, x_{n}\right)$ denotes that the matrix $\mathbf{Q}(\mathbf{x})$ is a diagonal matrix whose diagonal elements are $x_{1}, \ldots, x_{n}$.

\section{B. Positive T-S Fuzzy Model with Time Delay}

The dynamics of the positive T-S fuzzy system with time delay is given as follows:

Rule $i$ : IF $\theta_{1}(t)$ is $M_{1}^{i}$ AND $\cdots$ AND $\theta_{\Psi}(t)$ is $M_{\Psi}^{i}$ THEN

$$
\left\{\begin{array}{l}
\dot{\mathbf{x}}(t)=\mathbf{A}_{i} \mathbf{x}(t)+\mathbf{A}_{\omega i} \tilde{\mathbf{w}}(t)+\mathbf{A}_{d i} \mathbf{x}(t-\tau) \\
\mathbf{y}(t)=\mathbf{C}_{i} \mathbf{x}(t)+\mathbf{C}_{\omega i} \tilde{\mathbf{w}}(t)+\mathbf{C}_{d i} \mathbf{x}(t-\tau) \\
\mathbf{z}(t)=\mathbf{E}_{i} \mathbf{x}(t)+\mathbf{E}_{\omega i} \tilde{\mathbf{w}}(t)+\mathbf{E}_{d i} \mathbf{x}(t-\tau) \\
\mathbf{x}(\varrho)=\chi(\varrho), \varrho \in[-\tau, 0]
\end{array}\right.
$$

where $\theta_{l}(t)$ is the premise variable, $\Psi$ is a positive integer, $l \in\{1,2, \ldots, \Psi\} ; M_{l}^{i}$ is the fuzzy set of the $i$-th rule corresponding to the function $\theta_{l}(t) ; \mathbf{x}(t) \in \Re^{n}, \tilde{\mathbf{w}}(t) \in \Re^{m}$, $\mathbf{y}(t) \in \Re^{l}$ and $\mathbf{z}(t) \in \Re^{q}$ are the system state vector, the disturbance signal, measurement output and the signal to be estimated, respectively; $\tau>0$ is a given constant time-delay and $\chi(\varrho)$ is the initial function; $\mathbf{A}_{i}, \mathbf{A}_{\omega i}, \mathbf{A}_{d i}, \mathbf{C}_{i}, \mathbf{C}_{\omega i}$, $\mathbf{C}_{d i}, \mathbf{E}_{i}, \mathbf{E}_{\omega i} \mathbf{E}_{d i}$ are the system matrices with compatible dimensions.

We get the dynamics of the positive T-S fuzzy system with time delay as follows:

$$
\left\{\begin{array}{l}
\dot{\mathbf{x}}(t)=\sum_{i=1}^{p} \mu_{i}(\theta(t))\left(\mathbf{A}_{i} \mathbf{x}(t)+\mathbf{A}_{\omega i} \tilde{\mathbf{w}}(t)+\mathbf{A}_{d i} \mathbf{x}(t-\tau)\right), \\
\mathbf{y}(t)=\sum_{i=1}^{p} \mu_{i}(\theta(t))\left(\mathbf{C}_{i} \mathbf{x}(t)+\mathbf{C}_{\omega i} \tilde{\mathbf{w}}(t)+\mathbf{C}_{d i} \mathbf{x}(t-\tau)\right), \\
\mathbf{z}(t)=\sum_{i=1}^{p} \mu_{i}(\theta(t))\left(\mathbf{E}_{i} \mathbf{x}(t)+\mathbf{E}_{\omega i} \tilde{\mathbf{w}}(t)+\mathbf{E}_{d i} \mathbf{x}(t-\tau)\right), \\
\mathbf{x}(\varrho)=\chi(\varrho), \varrho \in[-\tau, 0],
\end{array}\right.
$$

where $\mu_{i}(\theta(t))=\frac{\prod_{l=1}^{\Psi} \phi_{M_{l}^{i}}(\theta(t))}{\sum_{k=1}^{p} \prod_{l=1}^{\Psi} \phi_{M_{l}^{k}}(\theta(t))}, \sum_{i=1}^{p} \mu_{i}(\theta(t))=1$, $\mu_{i}(\theta(t)) \geq 0, \forall i$ and $\mu_{i}(\theta(t))$ is the normalized grade of mem- bership; $\phi_{M_{l}^{i}}(\theta(t))$ is the grade of membership corresponding to the fuzzy term $M_{l}^{i} ; p$ is the number of the fuzzy rules.

The following assumptions and definitions are presented to support the analysis.

Assumption 1: All of the system states of a positive nonlinear system are not able to be measured.

Assumption 2: A positive nonlinear system is affected by disturbance signals and time delays.

Assumption 3: A positive nonlinear system is able to be expressed by a positive T-S fuzzy model (2).

Definition 1: [18] A system is deemed to be positive if the initial condition $\mathbf{x}(0)=\mathbf{x}_{0} \succeq 0$ holds and the corresponding trajectory $\mathbf{x}(t) \succeq 0, \mathbf{z}(t) \succeq 0, \mathbf{y}(t) \succeq 0$ and $\tilde{\mathbf{w}}(t) \succeq 0$ for all $t \geq 0$ is satisfied.

Definition 2: [1] A matrix $\mathbf{M}$ is called a Metzler matrix if its off-diagonal elements are non-negative: $m_{r s} \succeq 0, r \neq s$.

Lemma 1: [56], [57] System (2) is a positive system if $\mathbf{A}_{i}$ is a Metzler matrix, and the rest of the system matrices satisfy that all of the elements in each matrix are non-negative.

\section{Positive T-S Fuzzy Filter Design}

The positive T-S fuzzy filter is described by $c$ rules of the following format:

$$
\begin{aligned}
& \text { Rule } j \text { : IF } \vartheta_{1}(t) \text { is } N_{1}^{j} \text { AND } \cdots \text { AND } \vartheta_{\Omega}(t) \text { is } N_{\Omega}^{j} \\
& \text { THEN } \\
& \left\{\begin{array}{l}
\dot{\mathbf{x}}_{f}(t)=\mathbf{A}_{f j} \mathbf{x}_{f}(t)+\mathbf{B}_{f j} \mathbf{y}(t), \\
\mathbf{z}_{f}(t)=\mathbf{C}_{f j} \mathbf{x}_{f}(t)+\mathbf{D}_{f j} \mathbf{y}(t),
\end{array}\right.
\end{aligned}
$$

where $\mathbf{x}_{f}(t) \in \Re^{n}$ is the filter state, $\mathbf{z}_{f}(t) \in \Re^{q}$ is the estimated output, $\mathbf{A}_{f j}, \mathbf{B}_{f j}, \mathbf{C}_{f j}, \mathbf{D}_{f j}$ are the positive T-S fuzzy filter gain matrices to be determined.

Then the overall positive T-S fuzzy filter is established as follows:

$$
\left\{\begin{aligned}
\dot{\mathbf{x}}_{f}(t) & =\sum_{j=1}^{c} \eta_{j}(\vartheta(t))\left(\mathbf{A}_{f j} \mathbf{x}_{f}(t)+\mathbf{B}_{f j} \mathbf{y}(t)\right) \\
\mathbf{z}_{f}(t) & =\sum_{j=1}^{c} \eta_{j}(\vartheta(t))\left(\mathbf{C}_{f j} \mathbf{x}(t)+\mathbf{D}_{f j} \mathbf{y}(t)\right)
\end{aligned}\right.
$$

where $\eta_{j}(\vartheta(t))=\frac{\prod_{\beta=1}^{\Omega} \phi_{N_{\beta}^{j}}(\vartheta(t))}{\sum_{k=1}^{c} \prod_{\beta=1}^{\Omega} \phi_{N_{\beta}^{k}}(\vartheta(t))}, \eta_{j}(\vartheta(t)) \geq 0$, $\sum_{j=1}^{c} \eta_{j}(\vartheta(t))=1, \forall j$ and $\eta_{j}(\vartheta(t))$ is the normalized grade of membership; $\phi_{N_{\beta}^{j}}(\vartheta(t))$ is the grade of membership corresponding to the fuzzy term of $N_{\beta}^{j}$.

For the sake of simplicity, we will omit $t$ in the following analysis. That means $\mathbf{x}(t), \mathbf{x}(t-\tau), \mathbf{x}_{f}(t), \mathbf{z}(t), \mathbf{z}_{f}(t), \mathbf{y}(t)$ and $\tilde{\mathbf{w}}(t)$ will be replaced by $\mathbf{x}, \mathbf{x}_{\tau}, \mathbf{x}_{f}, \mathbf{z}, \mathbf{z}_{f}, \mathbf{y}$ and $\tilde{\mathbf{w}}$, respectively. Meanwhile, $\mu_{i}(\theta(t))$ and $\eta_{j}(\vartheta(t))$ will be replaced by $\mu_{i}$ and $\eta_{j}$, respectively.

Defining $\zeta=\left[\mathbf{x} ; \mathbf{x}_{f}-\mathbf{x}\right]$ and $\tilde{\mathbf{z}}=\mathbf{z}_{f}-\mathbf{z}$, the positive T-S fuzzy filter error system is written as follows:

$$
\left\{\begin{aligned}
\dot{\zeta} & =\sum_{i=1}^{p} \sum_{j=1}^{c} \mu_{i} \eta_{j}\left(\hat{\mathbf{A}}_{i j} \zeta+\hat{\mathbf{A}}_{\omega i j} \tilde{\mathbf{w}}+\hat{\mathbf{A}}_{d i j} \mathbf{x}_{\tau}\right), \\
\tilde{\mathbf{z}} & =\sum_{i=1}^{p} \sum_{j=1}^{c} \mu_{i} \eta_{j}\left(\hat{\mathbf{L}}_{i j} \zeta+\hat{\mathbf{L}}_{\omega i j} \tilde{\mathbf{w}}+\hat{\mathbf{L}}_{d i j} \mathbf{x}_{\tau}\right),
\end{aligned}\right.
$$


where $\quad \hat{\mathbf{A}}_{i j}=\left[\begin{array}{cc}\mathbf{A}_{i} & \mathbf{0} \\ \mathbf{A}_{f j}+\mathbf{B}_{f j} \mathbf{C}_{i}-\mathbf{A}_{i} & \mathbf{A}_{f j}\end{array}\right], \quad \hat{\mathbf{A}}_{\omega i j}=$ $\left[\begin{array}{c}\mathbf{A}_{\omega i} \\ \mathbf{B}_{f j} \mathbf{C}_{\omega i}-\mathbf{A}_{\omega i}\end{array}\right], \quad \hat{\mathbf{A}}_{d i j}=\left[\begin{array}{c}\mathbf{A}_{d i} \\ \mathbf{B}_{f j} \mathbf{C}_{d i}-\mathbf{A}_{d i}\end{array}\right], \quad \hat{\mathbf{L}}_{i j}=$ $\left[\begin{array}{ll}\mathbf{D}_{f j} \mathbf{C}_{i}-\mathbf{E}_{i}+\mathbf{C}_{f j} & \mathbf{C}_{f j}\end{array}\right], \quad \hat{\mathbf{L}}_{\omega i j}=\left[\mathbf{D}_{f j} \mathbf{C}_{\omega i}-\mathbf{E}_{\omega i}\right]$. $\hat{\mathbf{L}}_{d i j}=\left[\mathbf{D}_{f j} \mathbf{C}_{d i}-\mathbf{E}_{d i}\right]$.

Remark 1: On the strength of Lemma 1, the positive T-S fuzzy filter (4) is positive, if $\mathbf{A}_{f j}$ is a Metzler matrix, $\mathbf{B}_{f j} \succeq 0$, $\mathbf{C}_{f j} \succeq 0, \mathbf{D}_{f j} \succeq 0$ for all $j$.

Remark 2: Based on Lemma 1, the positive T-S fuzzy filter error system (5) is positive, if $\hat{\mathbf{A}}_{i j}$ is a Metzler matrix, $\hat{\mathbf{A}}_{\omega i j} \succeq$ $0, \hat{\mathbf{A}}_{d i j} \succeq 0, \hat{\mathbf{L}}_{i j} \succeq 0, \hat{\mathbf{L}}_{\omega i j} \succeq 0, \hat{\mathbf{L}}_{d i j} \succeq 0$, for all $i, j$.

For further investigating the filter design, the definition of $L_{1}$-induced performance is given firstly in the following.

Definition 3: [58] It is seen that the system (5) has $L_{1}$ induced performance at the level $\gamma$, if under zero initial conditions, the following inequality is satisfied

$$
\|\tilde{\mathbf{z}}\|_{L_{1}}<\gamma|| \tilde{\mathbf{w}} \|_{L_{1}}
$$

where $\gamma$ is the optimal level to be determined.

In this paper, for a given stable positive T-S fuzzy system with time delay, we aim to design a positive T-S fuzzy filter so that the stability and positivity of the positive T-S fuzzy filter error system can be ensured under $L_{1}$-induced performance. Therefore, let us focus on this target in the following work.

\section{Stability AnAlysis}

In this section, to facilitate the stability and positivity analysis, a novel augmented positive T-S fuzzy filter error system is given by introducing an auxiliary variable. Furthermore, to better extract the positivity of the positive system, a linear co-positive Lyapunov function is used to analyze the stability and positivity of the positive T-S fuzzy filter error system. In addition, the advanced PLMFs technique is considered to help reduce the conservativeness of the results.

\section{A. The Augmented Positive T-S Fuzzy Filter Error System}

In the matrices $\hat{\mathbf{A}}_{i j}, \hat{\mathbf{A}}_{\omega i j}, \hat{\mathbf{A}}_{d i j}, \hat{\mathbf{L}}_{i j}, \hat{\mathbf{L}}_{\omega i j}$ and $\hat{\mathbf{L}}_{d i j}$, we can find the determined matrices $\mathbf{B}_{f j}, \mathbf{D}_{f j}$ are coupled with $\mathbf{C}_{i}, \mathbf{C}_{\omega i}$, and $\mathbf{C}_{d i}$, which will make the non-convex terms in stability conditions is hard to deal with. In order to solve this problem, we try to pick out these determined matrices from $\hat{\mathbf{A}}_{i j}, \hat{\mathbf{A}}_{\omega i j}, \hat{\mathbf{A}}_{d i j}, \hat{\mathbf{L}}_{i j}, \hat{\mathbf{L}}_{\omega i j}$ and $\hat{\mathbf{L}}_{d i j}$. Hence, an auxiliary variable which is shown as (8) is adopted to facilitate the analysis. Then the system matrices of the positive T-S fuzzy filter error system are dealt with as follows:

$$
\begin{aligned}
& \hat{\mathbf{A}}_{i j}=\overline{\mathbf{A}}_{i j}+\mathbf{M}_{j} \tilde{\mathbf{H}}_{i} \tilde{\mathbf{C}}, \hat{\mathbf{A}}_{\omega i j}=\overline{\mathbf{A}}_{\omega i}+\mathbf{M}_{j} \tilde{\mathbf{H}}_{i} \tilde{\mathbf{D}}, \\
& \hat{\mathbf{A}}_{d i j}=\overline{\mathbf{A}}_{d i}+\mathbf{M}_{j} \tilde{\mathbf{H}}_{i} \tilde{\mathbf{C}}_{d}, \hat{\mathbf{L}}_{i j}=\overline{\mathbf{E}}_{i j}+\mathbf{N}_{j} \tilde{\mathbf{H}}_{i} \tilde{\mathbf{C}}, \\
& \hat{\mathbf{L}}_{\omega i j}=\overline{\mathbf{E}}_{\omega i}+\mathbf{N}_{j} \tilde{\mathbf{H}}_{i} \tilde{\mathbf{D}}, \hat{\mathbf{L}}_{d i j}=\overline{\mathbf{E}}_{d i}+\mathbf{N}_{j} \tilde{\mathbf{H}}_{i} \tilde{\mathbf{C}}_{d} .
\end{aligned}
$$

where $\overline{\mathbf{A}}_{i j}=\left[\begin{array}{cc}\mathbf{A}_{i} & \mathbf{0} \\ \mathbf{A}_{f j}-\mathbf{A}_{i} & \mathbf{A}_{f j}\end{array}\right], \overline{\mathbf{A}}_{\omega i}=\left[\begin{array}{c}\mathbf{A}_{\omega i} \\ -\mathbf{A}_{\omega i}\end{array}\right], \overline{\mathbf{A}}_{d i}=$ $\left[\begin{array}{c}\mathbf{A}_{d i} \\ -\mathbf{A}_{d i}\end{array}\right], \tilde{\mathbf{C}}=\left[\begin{array}{ll}\mathbf{I} & \mathbf{0} \\ \mathbf{0} & \mathbf{0} \\ \mathbf{0} & \mathbf{0}\end{array}\right], \tilde{\mathbf{D}}=\left[\begin{array}{l}\mathbf{0} \\ \mathbf{I} \\ \mathbf{0}\end{array}\right], \tilde{\mathbf{C}}_{d}=\left[\begin{array}{l}\mathbf{0} \\ \mathbf{0} \\ \mathbf{I}\end{array}\right], \tilde{\mathbf{H}}_{i}=$
$\left[\begin{array}{lll}\mathbf{C}_{i} & \mathbf{C}_{\omega i} & \mathbf{C}_{d i}\end{array}\right], \mathbf{M}_{j}=\left[\begin{array}{c}\mathbf{0} \\ \mathbf{B}_{f j}\end{array}\right], \mathbf{N}_{j}=\left[\mathbf{D}_{f j}\right], \overline{\mathbf{E}}_{i j}=$ $\left[\begin{array}{ll}\mathbf{C}_{f j}-\mathbf{E}_{i} & \mathbf{C}_{f j}\end{array}\right], \overline{\mathbf{E}}_{\omega i}=\left[-\mathbf{E}_{\omega i}\right], \overline{\mathbf{E}}_{d i}=\left[-\mathbf{E}_{d i}\right]$.

The auxiliary variable is chosen as follows:

$$
\tilde{\psi}=\sum_{i=1}^{p} \mu_{i}\left(\tilde{\mathbf{H}}_{i} \tilde{\mathbf{C}} \zeta+\tilde{\mathbf{H}}_{i} \tilde{\mathbf{D}} \tilde{\mathbf{w}}+\tilde{\mathbf{H}}_{i} \tilde{\mathbf{C}}_{d} \mathbf{x}_{\tau}\right) .
$$

Due to $\tilde{\mathbf{H}}_{i} \succeq 0, \tilde{\mathbf{C}} \succeq 0, \tilde{\mathbf{D}} \succeq 0$ and $\tilde{\mathbf{C}}_{d} \succeq 0$, we can see that each element in $\tilde{\psi}$ is non-negative. Then following on (5) and (8), an augmented system is presented with the defination of $\mathbf{E}=\left[\begin{array}{lr}\mathbf{I} & \mathbf{0} \\ \mathbf{0} & \mathbf{0}\end{array}\right], \xi=[\zeta ; \tilde{\psi}]$ :

$$
\begin{aligned}
& \mathbf{E} \dot{\xi}=\sum_{i=1}^{p} \sum_{j=1}^{c} \mu_{i} \eta_{j}\left(\tilde{\mathbf{A}}_{i j} \xi+\tilde{\mathbf{A}}_{\omega i} \tilde{\mathbf{w}}+\tilde{\mathbf{A}}_{d i} \mathbf{x}_{\tau}\right), \\
& \tilde{\mathbf{z}}=\sum_{i=1}^{p} \sum_{j=1}^{c} \mu_{i} \eta_{j}\left(\tilde{\mathbf{L}}_{i j} \xi+\tilde{\mathbf{L}}_{\omega i} \tilde{\mathbf{w}}+\tilde{\mathbf{L}}_{d i} \mathbf{x}_{\tau}\right),
\end{aligned}
$$

where $\tilde{\mathbf{A}}_{i j}=\left[\begin{array}{cc}\overline{\mathbf{A}}_{i j} & \mathbf{M}_{j} \\ \tilde{\mathbf{H}}_{i} \tilde{\mathbf{C}} & -\mathbf{I}\end{array}\right], \tilde{\mathbf{A}}_{\omega i}=\left[\begin{array}{c}\overline{\mathbf{A}}_{\omega i} \\ \tilde{\mathbf{H}}_{i} \tilde{\mathbf{D}}\end{array}\right], \tilde{\mathbf{A}}_{d i}=\left[\begin{array}{c}\overline{\mathbf{A}}_{d i} \\ \tilde{\mathbf{H}}_{i} \tilde{\mathbf{C}}_{d}\end{array}\right]$, $\tilde{\mathbf{L}}_{i j}=\left[\begin{array}{ll}\overline{\mathbf{E}}_{i j} & \mathbf{N}_{j}\end{array}\right], \tilde{\mathbf{L}}_{\omega i}=\left[\overline{\mathbf{E}}_{\omega i}\right], \tilde{\mathbf{L}}_{d i}=\left[\overline{\mathbf{E}}_{d i}\right]$.

\section{B. Positivity and Stability Analysis of Positive T-S Fuzzy Filter Error System}

In the following, a proper Lyapunov function is chosen so that not only can the positivity of the positive system be captured, but also the time delay can be taken into account:

$$
V(t)=\mathbf{p}^{T} \mathbf{E} \xi+\int_{t-\tau}^{t} \lambda^{T} \mathbf{F}_{d} \mathbf{x}(s) d_{s},
$$

where $0 \preceq \lambda \in \Re^{n}$ is a vector to be determined and $0 \preceq \mathbf{F}_{d} \in$ $\Re^{n \times n}$ is a given matrix.

The derivative of the above Lyapunov function is obtained:

$$
\begin{aligned}
\dot{V}(t)= & \mathbf{p}^{T} \mathbf{E} \dot{\xi}+\lambda^{T} \mathbf{F}_{d} \mathbf{x}-\lambda^{T} \mathbf{F}_{d} \mathbf{x}_{\tau} \\
= & \mathbf{p}^{T} \sum_{i=1}^{p} \sum_{j=1}^{c} \mu_{i} \eta_{j}\left(\tilde{\mathbf{A}}_{i j} \xi+\tilde{\mathbf{A}}_{\omega i} \tilde{\mathbf{w}}+\tilde{\mathbf{A}}_{d i} \mathbf{x}_{\tau}\right) \\
& +\lambda^{T} \mathbf{F}_{d} \mathbf{x}-\lambda^{T} \mathbf{F}_{d} \mathbf{x}_{\tau} .
\end{aligned}
$$

Next, taking the $L_{1}$ performance index (6) into account:

$$
\begin{aligned}
J & =\int_{0}^{T}\|\tilde{\mathbf{z}}\|_{L_{1}}-\gamma\|\tilde{\mathbf{w}}\|_{L_{1}} d_{t}=\int_{0}^{T} \sum_{k=1}^{q} \tilde{z}_{k}-\gamma \sum_{k=1}^{m} \tilde{w}_{k} d_{t} \\
& =\int_{0}^{T} \sum_{k=1}^{q} \tilde{z}_{k}-\gamma \sum_{k=1}^{m} \tilde{w}_{k}+\dot{V}(t) d_{t}+V(0)-V(T) .
\end{aligned}
$$

Due to the zero initial condition, and $V(T) \rightarrow 0$ when $T \rightarrow \infty,(12)$ can be treated as:

$$
\begin{aligned}
J & =\int_{0}^{\infty} \sum_{k=1}^{q} \tilde{z}_{k}-\gamma \sum_{k=1}^{m} \tilde{w}_{k}+\dot{V}(t) d_{t} \\
& =\int_{0}^{\infty} \mathbf{I}_{1}^{T} \tilde{\mathbf{z}}-\gamma \mathbf{I}_{2}^{T} \tilde{\mathbf{w}}+\dot{V}(t) d_{t},
\end{aligned}
$$

where $\mathbf{I}_{1} \in \Re^{q}$ and $\mathbf{I}_{2} \in \Re^{m}$ are vectors with all of the elements being 1 . 
Taking (9) and (11) into (13), we have:

$$
\begin{aligned}
J= & \int_{0}^{\infty} \mathbf{I}_{1}^{T}\left(\sum_{i=1}^{p} \sum_{j=1}^{c} \mu_{i} \eta_{j}\left(\tilde{\mathbf{L}}_{i j} \xi+\tilde{\mathbf{L}}_{\omega i} \tilde{\mathbf{w}}+\tilde{\mathbf{L}}_{d i} \mathbf{x}_{\tau}\right)\right) \\
& +\mathbf{p}^{T}\left(\sum_{i=1}^{p} \sum_{j=1}^{c} \mu_{i} \eta_{j}\left(\tilde{\mathbf{A}}_{i j} \xi+\tilde{\mathbf{A}}_{\omega i} \tilde{\mathbf{w}}+\tilde{\mathbf{A}}_{d i} \mathbf{x}_{\tau}\right)\right) \\
& +\lambda^{T} \mathbf{F}_{d} \mathbf{x}-\lambda^{T} \mathbf{F}_{d} \mathbf{x}_{\tau}-\gamma \mathbf{I}_{2}^{T} \tilde{\mathbf{w}} d_{t} \\
= & \int_{0}^{\infty} \sum_{i=1}^{p} \sum_{j=1}^{c} \mu_{i} \eta_{j}\left(\left(\mathbf{I}_{1}^{T} \tilde{\mathbf{L}}_{\omega i}+\mathbf{p}^{T} \tilde{\mathbf{A}}_{\omega i}-\gamma \mathbf{I}_{2}^{T}\right) \tilde{\mathbf{w}}\right. \\
& +\left(\mathbf{I}_{1}^{T} \tilde{\mathbf{L}}_{i j}+\mathbf{p}^{T} \tilde{\mathbf{A}}_{i j}+\lambda^{T} \mathbf{F}_{d}\left[\mathbf{I}_{n \times n} \quad \mathbf{0}_{n \times(n+l)}\right]\right) \xi \\
& \left.+\left(\mathbf{I}_{1}^{T} \tilde{\mathbf{L}}_{d i}+\mathbf{p}^{T} \tilde{\mathbf{A}}_{d i}-\lambda^{T} \mathbf{F}_{d}\right) \mathbf{x}_{\tau}\right) d_{t},
\end{aligned}
$$

where $\mathbf{I}_{n \times n}$ is an unit matrix with the dimensions of $n \times n$, $\mathbf{0}_{n \times(n+l)}$ is a null matrix with the dimensions of $n \times(n+l)$.

Based on (14), we can see that $J<0$ can be ensured by the following conditions:

$$
\begin{aligned}
& \mathbf{I}_{1}^{T} \tilde{\mathbf{L}}_{i j}+\mathbf{p}^{T} \tilde{\mathbf{A}}_{i j}+\lambda^{T} \mathbf{F}_{d}\left[\mathbf{I}_{n \times n} \quad \mathbf{0}_{n \times(n+l)}\right] \prec 0, \forall i, j ; \\
& \mathbf{I}_{1}^{T} \tilde{\mathbf{L}}_{\omega i}+\mathbf{p}^{T} \tilde{\mathbf{A}}_{\omega i}-\gamma \mathbf{I}_{2}^{T} \prec 0, \forall i ; \\
& \mathbf{I}_{1}^{T} \tilde{\mathbf{L}}_{d i}+\mathbf{p}^{T} \tilde{\mathbf{A}}_{d i}-\lambda^{T} \mathbf{F}_{d} \prec 0, \forall i .
\end{aligned}
$$

For further handling the above inequalities, we have:

$$
\begin{aligned}
& \mathbf{I}_{1}^{T} \tilde{\mathbf{L}}_{i j}+\mathbf{p}^{T} \tilde{\mathbf{A}}_{i j}+\lambda^{T} \mathbf{F}_{d}\left[\mathbf{I}_{n \times n} \quad \mathbf{0}_{n \times(n+l)}\right] \\
& =\mathbf{I}_{1}^{T}\left[\begin{array}{ll}
\overline{\mathbf{E}}_{i j} & \mathbf{N}_{j}
\end{array}\right]+\left[\begin{array}{ll}
\mathbf{p}_{1}^{T} & \mathbf{p}_{2}^{T}
\end{array}\right]\left[\begin{array}{cc}
\overline{\mathbf{A}}_{i j} & \mathbf{M}_{j} \\
\tilde{\mathbf{H}}_{i} \tilde{\mathbf{C}} & -\mathbf{I}
\end{array}\right] \\
& +\lambda^{T} \mathbf{F}_{d}\left[\begin{array}{ll}
\mathbf{I}_{n \times n} & \mathbf{0}_{n \times(n+l)}
\end{array}\right] \\
& =\left[\mathbf{I}_{1}^{T} \overline{\mathbf{E}}_{i j}+\mathbf{p}_{1}^{T} \overline{\mathbf{A}}_{i j}+\mathbf{p}_{2}^{T} \tilde{\mathbf{H}}_{i} \tilde{\mathbf{C}}+\lambda^{T} \mathbf{F}_{d}\left[\begin{array}{|ll}
\mathbf{I}_{n \times n} & \mathbf{0}_{n \times n}
\end{array}\right]\right. \\
& \left.\mathbf{I}_{1}^{T} \mathbf{N}_{j}+\mathbf{p}_{1}^{T} \mathbf{M}_{j}-\mathbf{p}_{2}^{T}\right], \\
& \mathbf{I}_{1}^{T} \tilde{\mathbf{L}}_{\omega i}+\mathbf{p}^{T} \tilde{\mathbf{A}}_{\omega i}-\gamma \mathbf{I}_{2}^{T} \\
& =\mathbf{I}_{1}^{T} \overline{\mathbf{E}}_{\omega i}-\gamma \mathbf{I}_{2}^{T}+\left[\begin{array}{ll}
\mathbf{p}_{1}^{T} & \mathbf{p}_{2}^{T}
\end{array}\right]\left[\begin{array}{c}
\overline{\mathbf{A}}_{\omega i} \\
\tilde{\mathbf{H}}_{i} \tilde{\mathbf{D}}
\end{array}\right] \\
& =\mathbf{I}_{1}^{T} \overline{\mathbf{E}}_{\omega i}-\gamma \mathbf{I}_{2}^{T}+\mathbf{p}_{1}^{T} \overline{\mathbf{A}}_{\omega i}+\mathbf{p}_{2}^{T} \tilde{\mathbf{H}}_{i} \tilde{\mathbf{D}}, \\
& \mathbf{I}_{1}^{T} \tilde{\mathbf{L}}_{d i}+\mathbf{p}^{T} \tilde{\mathbf{A}}_{d i}-\lambda^{T} \mathbf{F}_{d} \\
& =\mathbf{I}_{1}^{T} \overline{\mathbf{E}}_{d i}+\left[\begin{array}{ll}
\mathbf{p}_{1}^{T} & \mathbf{p}_{2}^{T}
\end{array}\right]\left[\begin{array}{c}
\overline{\mathbf{A}}_{d i} \\
\tilde{\mathbf{H}}_{i} \tilde{\mathbf{C}}_{d}
\end{array}\right]-\lambda^{T} \mathbf{F}_{d} \\
& =\mathbf{I}_{1}^{T} \overline{\mathbf{E}}_{d i}+\mathbf{p}_{1}^{T} \overline{\mathbf{A}}_{d i}+\mathbf{p}_{2}^{T} \tilde{\mathbf{H}}_{i} \tilde{\mathbf{C}}_{d}-\lambda^{T} \mathbf{F}_{d} .
\end{aligned}
$$

Through substituting the expressions of $\overline{\mathbf{A}}_{i j}, \overline{\mathbf{A}}_{\omega i}, \overline{\mathbf{A}}_{d i}, \overline{\mathbf{E}}_{i j}$, $\overline{\mathbf{E}}_{\omega i}, \overline{\mathbf{E}}_{d i}, \tilde{\mathbf{C}}, \tilde{\mathbf{D}}, \tilde{\mathbf{C}}_{d}, \tilde{\mathbf{H}}_{i}, \mathbf{M}_{j}$ and $\mathbf{N}_{j}$ into the above equalities, the following conditions hold:

$$
\begin{aligned}
& \mathbf{I}_{1}^{T} \overline{\mathbf{E}}_{i j}+\mathbf{p}_{1}^{T} \overline{\mathbf{A}}_{i j}+\mathbf{p}_{2}^{T} \tilde{\mathbf{H}}_{i} \tilde{\mathbf{C}}+\lambda^{T} \mathbf{F}_{d}\left[\mathbf{I}_{n \times n} \quad \mathbf{0}_{n \times n}\right] \\
& =\mathbf{I}_{1}^{T}\left[\begin{array}{ll}
\mathbf{C}_{f j}-\mathbf{E}_{i} & \mathbf{C}_{f j}
\end{array}\right]+\left[\begin{array}{ll}
\mathbf{p}_{11}^{T} & \mathbf{p}_{12}^{T}
\end{array}\right]\left[\begin{array}{cc}
\mathbf{A}_{i} & \mathbf{0} \\
\mathbf{A}_{f j}-\mathbf{A}_{i} & \mathbf{A}_{f j}
\end{array}\right] \\
& +\mathbf{p}_{2}^{T}\left[\begin{array}{lll}
\mathbf{C}_{i} & \mathbf{C}_{\omega i} & \mathbf{C}_{d i}
\end{array}\right]\left[\begin{array}{ll}
\mathbf{I} & \mathbf{0} \\
\mathbf{0} & \mathbf{0} \\
\mathbf{0} & \mathbf{0}
\end{array}\right]+\lambda^{T} \mathbf{F}_{d}\left[\begin{array}{ll}
\mathbf{I}_{n \times n} & \mathbf{0}_{n \times n}
\end{array}\right] \\
& =\left[\mathbf{I}_{1}^{T}\left(\mathbf{C}_{f j}-\mathbf{E}_{i}\right)+\mathbf{p}_{11}^{T} \mathbf{A}_{i}+\mathbf{p}_{12}^{T}\left(\mathbf{A}_{f j}-\mathbf{A}_{i}\right)+\mathbf{p}_{2}^{T} \mathbf{C}_{i}+\lambda^{T} \mathbf{F}_{d}\right. \\
& \left.\mathbf{p}_{12}^{T} \mathbf{A}_{f j}+\mathbf{I}_{1}^{T} \mathbf{C}_{f j}\right] \text {, }
\end{aligned}
$$

$$
\begin{aligned}
& \mathbf{I}_{1}^{T} \mathbf{N}_{j}+\mathbf{p}_{1}^{T} \mathbf{M}_{j}-\mathbf{p}_{2}^{T} \\
& =\mathbf{I}_{1}^{T} \mathbf{D}_{f j}+\left[\begin{array}{ll}
\mathbf{p}_{11}^{T} & \mathbf{p}_{12}^{T}
\end{array}\right]\left[\begin{array}{c}
\mathbf{0} \\
\mathbf{B}_{f j}
\end{array}\right]-\mathbf{p}_{2}^{T} \\
& =\mathbf{I}_{1}^{T} \mathbf{D}_{f j}+\mathbf{p}_{12}^{T} \mathbf{B}_{f j}-\mathbf{p}_{2}^{T}, \\
& \mathbf{I}_{1}^{T} \overline{\mathbf{E}}_{\omega i}-\gamma \mathbf{I}_{2}^{T}+\mathbf{p}_{1}^{T} \overline{\mathbf{A}}_{\omega i}+\mathbf{p}_{2}^{T} \tilde{\mathbf{H}}_{i} \tilde{\mathbf{D}} \\
& =-\mathbf{I}_{1}^{T} \mathbf{E}_{\omega i}-\gamma \mathbf{I}_{2}^{T}+\left[\begin{array}{ll}
\mathbf{p}_{11}^{T} & \mathbf{p}_{12}^{T}
\end{array}\right]\left[\begin{array}{c}
\mathbf{A}_{\omega i} \\
-\mathbf{A}_{\omega i}
\end{array}\right] \\
& +\mathbf{p}_{2}^{T}\left[\begin{array}{lll}
\mathbf{C}_{i} & \mathbf{C}_{\omega i} & \mathbf{C}_{d i}
\end{array}\right]\left[\begin{array}{l}
\mathbf{0} \\
\mathbf{I} \\
\mathbf{0}
\end{array}\right] \\
& =-\mathbf{I}_{1}^{T} \mathbf{E}_{\omega i}-\gamma \mathbf{I}_{2}^{T}+\left(\mathbf{p}_{11}^{T}-\mathbf{p}_{12}^{T}\right) \mathbf{A}_{\omega i}+\mathbf{p}_{2}^{T} \mathbf{C}_{\omega i}, \\
& \mathbf{I}_{1}^{T} \overline{\mathbf{E}}_{d i}+\mathbf{p}_{1}^{T} \overline{\mathbf{A}}_{d i}+\mathbf{p}_{2}^{T} \tilde{\mathbf{H}}_{i} \tilde{\mathbf{C}}_{d}-\lambda^{T} \mathbf{F}_{d} \\
& =-\mathbf{I}_{1}^{T} \mathbf{E}_{d i}+\left[\begin{array}{ll}
\mathbf{p}_{11}^{T} & \mathbf{p}_{12}^{T}
\end{array}\right]\left[\begin{array}{c}
\mathbf{A}_{d i} \\
-\mathbf{A}_{d i}
\end{array}\right] \\
& +\mathbf{p}_{2}^{T}\left[\begin{array}{lll}
\mathbf{C}_{i} & \mathbf{C}_{\omega i} & \mathbf{C}_{d i}
\end{array}\right]\left[\begin{array}{l}
\mathbf{0} \\
\mathbf{0} \\
\mathbf{I}
\end{array}\right]-\lambda^{T} \mathbf{F}_{d} \\
& =-\mathbf{I}_{1}^{T} \mathbf{E}_{d i}+\left(\mathbf{p}_{11}^{T}-\mathbf{p}_{12}^{T}\right) \mathbf{A}_{d i}+\mathbf{p}_{2}^{T} \mathbf{C}_{d i}-\lambda^{T} \mathbf{F}_{d} .
\end{aligned}
$$

In light of the above analysis, we can obtain the stability conditions as follows:

$$
\begin{aligned}
& \mathbf{I}_{1}^{T}\left(\mathbf{C}_{f j}-\mathbf{E}_{i}\right)+\mathbf{p}_{11}^{T} \mathbf{A}_{i}+\mathbf{p}_{12}^{T}\left(\mathbf{A}_{f j}-\mathbf{A}_{i}\right) \\
& +\mathbf{p}_{2}^{T} \mathbf{C}_{i}+\lambda^{T} \mathbf{F}_{d} \prec 0, \\
& \mathbf{p}_{12}^{T} \mathbf{A}_{f j}+\mathbf{I}_{1}^{T} \mathbf{C}_{f j} \prec 0, \\
& \mathbf{I}_{1}^{T} \mathbf{D}_{f j}+\mathbf{p}_{12}^{T} \mathbf{B}_{f j}-\mathbf{p}_{2}^{T} \prec 0, \\
& -\mathbf{I}_{1}^{T} \mathbf{E}_{\omega i}-\gamma \mathbf{I}_{2}^{T}+\left(\mathbf{p}_{11}^{T}-\mathbf{p}_{12}^{T}\right) \mathbf{A}_{\omega i}+\mathbf{p}_{2}^{T} \mathbf{C}_{\omega i} \prec 0, \\
& -\mathbf{I}_{1}^{T} \mathbf{E}_{d i}+\left(\mathbf{p}_{11}^{T}-\mathbf{p}_{12}^{T}\right) \mathbf{A}_{d i}+\mathbf{p}_{2}^{T} \mathbf{C}_{d i}-\lambda^{T} \mathbf{F}_{d} \prec 0 .
\end{aligned}
$$

On the basis of the above inequalities, it can be seen that $\mathbf{p}_{12}^{T} \mathbf{A}_{f j}$ and $\mathbf{p}_{12}^{T} \mathbf{B}_{f j}$ are non-convex terms. To transform them into convex ones, $\mathbf{P}_{A f, j}$ and $\mathbf{P}_{B f, j}$ are considered as follows:

$$
\mathbf{P}_{A f, j}=\left[\mathbf{p}_{12 k}^{T} \mathbf{A}_{f j, k}\right], \mathbf{P}_{B f, j}=\left[\mathbf{p}_{12 k}^{T} \mathbf{B}_{f j, k}\right],
$$

where $\mathbf{A}_{f j, k}$ and $\mathbf{B}_{f j, k}$ are the $k$-th row of $\mathbf{A}_{f j}$ and $\mathbf{B}_{f j}$ respectively. $\mathbf{p}_{12 k}$ is the $k$-th element in $\mathbf{p}_{12}$.

For example, we assume:

$$
\begin{aligned}
& \mathbf{A}_{f j}=\left[\begin{array}{lll}
a_{f j, 11} & a_{f j, 12} & a_{f j, 13} \\
a_{f j, 21} & a_{f j, 22} & a_{f j, 23} \\
a_{f j, 31} & a_{f j, 32} & a_{f j, 33}
\end{array}\right], \\
& \mathbf{p}_{12}=\left[\begin{array}{lll}
p_{121} & p_{122} & p_{123}
\end{array}\right]^{T} .
\end{aligned}
$$

Then, we have:

$$
\mathbf{P}_{A f, j}=\left[\begin{array}{lll}
p_{121} a_{f j, 11} & p_{121} a_{f j, 12} & p_{121} a_{f j, 13} \\
p_{122} a_{f j, 21} & p_{122} a_{f j, 22} & p_{122} a_{f j, 23} \\
p_{123} a_{f j, 31} & p_{123} a_{f j, 32} & p_{123} a_{f j, 33}
\end{array}\right] .
$$

Thereby, it can be obtained $\mathbf{p}_{12}^{T} \mathbf{A}_{f j}=\sum_{k=1}^{n}\left[\mathbf{P}_{A f, j k}\right]$, where $\mathbf{P}_{A f, j k}$ is the $k$-th row of $\mathbf{P}_{A f, j}$.

Taking (30) into (25)-(27), the following conditions hold:

$$
\begin{aligned}
& \mathbf{I}_{1}^{T}\left(\mathbf{C}_{f j}-\mathbf{E}_{i}\right)+\mathbf{p}_{11}^{T} \mathbf{A}_{i}+\sum_{k=1}^{n}\left[\mathbf{P}_{A f, j k}\right]-\mathbf{p}_{12}^{T} \mathbf{A}_{i} \\
& +\mathbf{p}_{2}^{T} \mathbf{C}_{i}+\lambda^{T} \mathbf{F}_{d} \prec 0,
\end{aligned}
$$




$$
\begin{aligned}
& \mathbf{I}_{1}^{T} \mathbf{C}_{f j}+\sum_{k=1}^{n}\left[\mathbf{P}_{A f, j k}\right] \prec 0, \\
& \mathbf{I}_{1}^{T} \mathbf{D}_{f j}+\sum_{k=1}^{n}\left[\mathbf{P}_{B f, j k}\right]-\mathbf{p}_{2}^{T} \prec 0 .
\end{aligned}
$$

Now, we are at the point that the convex stability conditions have been obtained. However, different from general systems, the positivity of the positive T-S fuzzy filter error system should be ensured as well. Thus, in the following, the positivity analysis will be of concern.

As mentioned in Remark 1 and Remark 2, the positivity conditions are shown as follows:

$$
\begin{aligned}
& \mathbf{A}_{f j} \text { is Metzler, } \mathbf{B}_{f j} \succeq 0, \mathbf{C}_{f j}\left(\mathbf{x}_{f}\right) \succeq 0, \mathbf{D}_{f j} \succeq 0, \forall j, \\
& \hat{\mathbf{A}}_{i j} \text { is Metzler, } \hat{\mathbf{A}}_{\omega i j} \succeq 0, \hat{\mathbf{A}}_{d i j} \succeq 0, \\
& \hat{\mathbf{L}}_{i j} \succeq 0, \hat{\mathbf{L}}_{\omega i j} \succeq 0, \hat{\mathbf{L}}_{d i j} \succeq 0, \forall i, j
\end{aligned}
$$

Because the open-loop system is a positive system, therefore, the positive condition (37) can be ensured by satisfying the positive condition (36) and the following inequalities:

$$
\begin{aligned}
& \mathbf{A}_{f j}+\mathbf{B}_{f j} \mathbf{C}_{i}-\mathbf{A}_{i} \succeq 0,, \forall i, j, \\
& \mathbf{B}_{f j} \mathbf{C}_{\omega i}-\mathbf{A}_{\omega i} \succeq 0, \forall i, j, \\
& \mathbf{B}_{f j} \mathbf{C}_{d i}-\mathbf{A}_{d i} \succeq 0, \forall i, j, \\
& \mathbf{D}_{f j} \mathbf{C}_{i}-\mathbf{E}_{i}+\mathbf{C}_{f j} \succeq 0, \forall i, j, \\
& \mathbf{D}_{f j} \mathbf{C}_{\omega i}-\mathbf{E}_{\omega i} \succeq 0, \forall i, j, \\
& \mathbf{D}_{f j} \mathbf{C}_{d i}-\mathbf{E}_{d i} \succeq 0, \forall i, j .
\end{aligned}
$$

On account of $\mathbf{p}_{12} \succeq 0$, we multiply both sides of the (38) - (40) by $\mathbf{p}_{12}^{T}$, respectively, so as to get convex positivity conditions, which are presented as follows:

$$
\begin{aligned}
& \mathbf{P}_{A f, j}+\mathbf{P}_{B f, j} \mathbf{C}_{i}-\left[\mathbf{p}_{12 k}^{T} \mathbf{A}_{i, k}\right] \succeq 0, \forall i, j, \\
& \mathbf{P}_{B f, j} \mathbf{C}_{\omega i}-\left[\mathbf{p}_{12 k}^{T} \mathbf{A}_{\omega i, k}\right] \succeq 0, \forall i, j, \\
& \mathbf{P}_{B f, j} \mathbf{C}_{d i}-\left[\mathbf{p}_{12 k}^{T} \mathbf{A}_{d i, k}\right], \succeq 0, \forall i, j,
\end{aligned}
$$

where $\mathbf{A}_{i, k}, \mathbf{A}_{\omega i, k}$ and $\mathbf{A}_{d i, k}$ are the $k$-th row of $\mathbf{A}_{i}, \mathbf{A}_{\omega i}$ and $\mathbf{A}_{d i}$, respectively. $\mathbf{p}_{12 k}$ is the $k$-th element in $\mathbf{p}_{12}$.

For facilitating the analysis, we define:

$$
\begin{aligned}
\tilde{\mathbf{F}}_{1 i j} & =\mathbf{P}_{A f, j}+\mathbf{P}_{B f, j} \mathbf{C}_{i}-\left[\mathbf{p}_{12 k}^{T} \mathbf{A}_{i, k}\right] \\
\tilde{\mathbf{F}}_{2 i j} & =\mathbf{P}_{B f, j} \mathbf{C}_{\omega i}-\left[\mathbf{p}_{12 k}^{T} \mathbf{A}_{\omega i, k}\right] \\
\tilde{\mathbf{F}}_{3 i j} & =\mathbf{P}_{B f, j} \mathbf{C}_{d i}-\left[\mathbf{p}_{12 k}^{T} \mathbf{A}_{d i, k}\right] \\
\tilde{\mathbf{F}}_{4 i j} & =\mathbf{D}_{f j} \mathbf{C}_{i}-\mathbf{E}_{i}+\mathbf{C}_{f j} \\
\tilde{\mathbf{F}}_{5 i j} & =\mathbf{D}_{f j} \mathbf{C}_{\omega i}-\mathbf{E}_{\omega i} \\
\tilde{\mathbf{F}}_{6 i j} & =\mathbf{D}_{f j} \mathbf{C}_{d i}-\mathbf{E}_{d i} \\
\tilde{\mathbf{Q}}_{1 i j} & =\mathbf{I}_{1}^{T}\left(\mathbf{C}_{f j}-\mathbf{E}_{i}\right)+\mathbf{p}_{11}^{T} \mathbf{A}_{i}+\sum_{k=1}^{n}\left[\mathbf{P}_{A f, j k}\right]-\mathbf{p}_{12}^{T} \mathbf{A}_{i} \\
+\mathbf{p}_{2}^{T} & \mathbf{C}_{i}+\lambda^{T} \mathbf{F}_{d} \\
\tilde{\mathbf{Q}}_{2 j} & =\sum_{k=1}^{n}\left[\mathbf{P}_{A f, j k}\right]+\mathbf{I}_{1}^{T} \mathbf{C}_{f j} \\
\tilde{\mathbf{Q}}_{3 j} & =\mathbf{I}_{1}^{T} \mathbf{D}_{f j}+\sum_{k=1}^{n}\left[\mathbf{P}_{B f, j k}\right]-\mathbf{p}_{2}^{T}
\end{aligned}
$$

$$
\begin{aligned}
& \tilde{\mathbf{Q}}_{4 i}=-\mathbf{I}_{1}^{T} \mathbf{E}_{\omega i}-\gamma \mathbf{I}_{2}^{T}+\left(\mathbf{p}_{11}^{T}-\mathbf{p}_{12}^{T}\right) \mathbf{A}_{\omega i}+\mathbf{p}_{2}^{T} \mathbf{C}_{\omega i} \\
& \tilde{\mathbf{Q}}_{5 i}=-\mathbf{I}_{1}^{T} \mathbf{E}_{d i}+\left(\mathbf{p}_{11}^{T}-\mathbf{p}_{12}^{T}\right) \mathbf{A}_{d i}+\mathbf{p}_{2}^{T} \mathbf{C}_{d i}-\lambda^{T} \mathbf{F}_{d}
\end{aligned}
$$

Remark 3: It is worth noting that the obtained results are very conservative because of the absence of the information of MFs. In general, researchers tend to use some mature techniques, like PDC technique and free-weighting matrix approach, to cut down the conservativeness of results instead of utilizing the information of MFs because the MFs will make stability analysis complex and MFD analysis is still in its early research stage. Thereby, taking the information of MFs into account for filter design of positive T-S fuzzy systems with time delay is very challenging but meaningful. In the following work, we will try our best to investigate the relaxed stability and positivity analysis for positive T-S fuzzy filter error systems.

C. Relaxed Positivity and Stability Analysis by using Piecewise-Linear Membership Functions

In this section, the PLMFs approximating method which has been explained in detail in [53], [59] is considered to extract the shape and boundary information of the original MFs. Meanwhile, the useful information of MFs is carried by some slack matrices to the positivity and stability analysis for relaxing the results.

According to (14), if the PLMFs method is considered, we obtain that $J<0$ can be ensured by the following conditions:

$$
\begin{aligned}
& \sum_{i=1}^{p} \sum_{j=1}^{c} \mu_{i} \eta_{j}\left(\mathbf{I}_{1}^{T} \tilde{\mathbf{L}}_{i j}+\mathbf{p}^{T} \tilde{\mathbf{A}}_{i j}+\lambda^{T} \mathbf{F}_{d}\left[\begin{array}{ll}
\mathbf{I}_{n \times n} & \mathbf{0}_{n \times(n+l)}
\end{array}\right]\right) \\
& \prec 0, \forall i, j ; \\
& \sum_{i=1}^{p} \sum_{j=1}^{c} \mu_{i} \eta_{j}\left(\mathbf{I}_{1}^{T} \tilde{\mathbf{L}}_{\omega i}+\mathbf{p}^{T} \tilde{\mathbf{A}}_{\omega i}-\gamma \mathbf{I}_{2}^{T}\right) \prec 0, \forall i, j ; \\
& \sum_{i=1}^{p} \sum_{j=1}^{c} \mu_{i} \eta_{j}\left(\mathbf{I}_{1}^{T} \tilde{\mathbf{L}}_{d i}+\mathbf{p}^{T} \tilde{\mathbf{A}}_{d i}-\lambda^{T} \mathbf{F}_{d}\right) \prec 0, \forall i, j
\end{aligned}
$$

Next, combining with the analysis from (18) to (56), we can obtain the convex stability conditions with MFs as follows:

$$
\begin{aligned}
& \sum_{i=1}^{p} \sum_{j=1}^{c} \mu_{i} \eta_{j}\left(\mathbf{Q}_{1 i j}\right) \prec 0, \forall i, j ; \\
& \sum_{i=1}^{p} \sum_{j=1}^{c} \mu_{i} \eta_{j}\left(\mathbf{Q}_{2 j}\right) \prec 0, \forall j ; \\
& \sum_{i=1}^{p} \sum_{j=1}^{c} \mu_{i} \eta_{j}\left(\mathbf{Q}_{3 j}\right) \prec 0, \forall j ; \\
& \sum_{i=1}^{p} \sum_{j=1}^{c} \mu_{i} \eta_{j}\left(\mathbf{Q}_{4 i}\right) \prec 0, \forall i ; \\
& \sum_{i=1}^{p} \sum_{j=1}^{c} \mu_{i} \eta_{j}\left(\mathbf{Q}_{5 i}\right) \prec 0, \forall i .
\end{aligned}
$$

In order to cope with the cross term $\mu_{i} \eta_{j}$ in above stability conditions, the PLMFs method are introduced in the following. Because $\mu_{i}$ is related to $\mathbf{x}$ but $\eta_{j}$ is related to $\mathbf{x}_{f}$, hence, we 
define $\hat{\mathbf{x}}=\left[\mathbf{x} ; \mathbf{x}_{f}\right] \in \Re^{2 n}$, and $\hat{x}_{1}=x_{1}, \hat{x}_{2}=x_{2}, \cdots$, $\hat{x}_{n}=x_{n}, \hat{x}_{n+1}=x_{f 1}, \hat{x}_{n+2}=x_{f 2}, \cdots, \hat{x}_{2 n}=x_{f n}$. Then dividing the whole operate domain into $S$ sub-domians, where the sub-domian $s$ is characterized by $\hat{x}_{l s d \text { min }} \leq \hat{x}_{l s} \leq \hat{x}_{l s m a x}$, $l \in\{1,2, \cdots, 2 n\}$ and $s \in\{1,2, \cdots, S\}$.

On the basis of the above definition, the cross term $\mu_{i} \eta_{j}$ can be replaced by the following expression:

$$
\mu_{i} \eta_{j}=h_{i j s}(\hat{\mathbf{x}})+\Delta_{i j s}(\hat{\mathbf{x}}), \forall i, j, s,
$$

where $h_{i j s}(\hat{\mathbf{x}})$ is the approximated function and $\Delta_{i j s}(\hat{\mathbf{x}})$ is the approximation error.

The approximated function $h_{i j s}(\hat{\mathbf{x}})$ is expressed as follows:

$$
h_{i j s}(\hat{\mathbf{x}})=\sum_{i_{1}=1}^{2} \sum_{i_{2}=1}^{2} \cdots \sum_{i_{2 n}=1}^{2} V_{1 i_{1} s}\left(\hat{x}_{1}\right) V_{2 i_{2} s}\left(\hat{x}_{2}\right) \cdots V_{2 n i_{2 n} s}\left(\hat{x}_{2 n}\right)
$$$$
\times \mu_{i}\left(D_{i_{1} i_{2} \cdots i_{2 n} s}\right) \eta_{j}\left(D_{i_{1} i_{2} \cdots i_{2 n} s}\right) \forall i, j, s,
$$

where $D_{i_{1} i_{2} \cdots i_{2 n} s}=\left[\begin{array}{llll}\hat{x}_{1 i_{1}} & \hat{x}_{2 i_{2}} \cdots & \hat{x}_{2 n i_{2 n}}\end{array}\right] . \hat{x}_{l i_{l}}=\hat{x}_{l d m i n}$ if $i_{l}=1$, and $\hat{x}_{l i_{l}}=\hat{x}_{l d \max }$ if $i_{l}=2, \forall l, s . V_{l i_{l} s}\left(\hat{x}_{l}\right)$ satisfies $\sum_{i_{l}=1}^{2} V_{l i_{l} s}\left(\hat{x}_{l}\right)=1, \forall l, s$.

Introducing (65) and (66) into the stability conditions (60) to (64), the relaxed stability conditions will be obtained. Because the derivations of relaxed stability conditions for (60) to (64) are similar, hence, we only show the derivation of relaxed results for (60). (61) to (64) will follow the same analysis.

$$
\begin{aligned}
& \sum_{i=1}^{p} \sum_{j=1}^{c} \mu_{i} \eta_{j} \mathbf{Q}_{1 i j}=\sum_{i=1}^{p} \sum_{j=1}^{c}\left(h_{i j s}(\hat{\mathbf{x}})+\Delta_{i j s}(\hat{\mathbf{x}})\right) \mathbf{Q}_{1 i j} \\
= & \sum_{i=1}^{p} \sum_{j=1}^{c}\left(h_{i j s}(\hat{\mathbf{x}})+\Delta_{i j s}(\hat{\mathbf{x}})-\gamma_{i j s}+\gamma_{i j s}\right) \mathbf{Q}_{1 i j} \\
\preceq & \sum_{i=1}^{p} \sum_{j=1}^{c}\left(\left(h_{i j s}(\hat{\mathbf{x}})+\gamma_{i j s}\right) \mathbf{Q}_{1 i j}+\left(\beta_{i j s}-\gamma_{i j s}\right) \mathbf{Y}_{1 i j}\right) \\
= & \sum_{i=1}^{p} \sum_{j=1}^{c}\left(\left(\sum_{i_{1}=1}^{2} \sum_{i_{2}=1}^{2} \cdots \sum_{i_{2 n}=1}^{2} V_{1 i_{1} s}\left(\hat{x}_{1}\right) V_{2 i_{2} s}\left(\hat{x}_{2}\right)\right.\right. \\
& \left.\cdots V_{2 n i_{2 n} s}\left(\hat{x}_{2 n}\right) \mu_{i}\left(D_{i_{1} i_{2} \cdots i_{2 n} s}\right) \eta_{j}\left(D_{i_{1} i_{2} \cdots i_{2 n} s}\right)+\gamma_{i j s}\right) \mathbf{Q}_{1 i j} \\
& \left.+\left(\beta_{i j s}-\gamma_{i j s}\right) \mathbf{Y}_{1 i j}\right) \\
= & \sum_{i_{1}=1}^{2} \sum_{i_{2}=1}^{2} \cdots \sum_{i_{2 n}=1}^{2} V_{1 i_{1} s}\left(\hat{x}_{1}\right) V_{2 i_{2} s}\left(\hat{x}_{2}\right) \cdots V_{2 n i_{2 n} s}\left(\hat{x}_{2 n}\right) \\
& \left(\sum _ { i = 1 } ^ { p } \sum _ { j = 1 } ^ { c } \left(\left(\mu_{i}\left(D_{i_{1} i_{2} \cdots i_{2 n} s}\right) \eta_{j}\left(D_{i_{1} i_{2} \cdots i_{2 n} s}\right)+\gamma_{i j s}\right) \mathbf{Q}_{1 i j}\right.\right. \\
& \left.\left.+\left(\beta_{i j s}-\gamma_{i j s}\right) \mathbf{Y}_{1 i j}\right)\right), \forall i, j, d,
\end{aligned}
$$

where $\gamma_{i j s}$ and $\beta_{i j s}$ are the lower and upper bound of $\Delta_{i j s}(\hat{\mathbf{x}})$, respectively, with satisfying $\gamma_{i j s} \leq \Delta_{i j s}(\hat{\mathbf{x}}) \leq \beta_{i j s}$. $\mathbf{Y}_{1 i j}$ is the slack matrix with satisfying $\mathbf{Y}_{1 i j} \succeq 0, \mathbf{Y}_{1 i j} \succeq \mathbf{Q}_{1 i j}$.

Similarly, by introducing the slack matrices $\mathbf{Y}_{2 j}, \mathbf{Y}_{3 j}, \mathbf{Y}_{4 i}$ and $\mathbf{Y}_{5 i}$ with satisfying $\mathbf{Y}_{2 j} \succeq 0, \mathbf{Y}_{2 j} \succeq \mathbf{Q}_{2 j}, \mathbf{Y}_{3 j} \succeq 0$, $\mathbf{Y}_{3 j} \succeq \mathbf{Q}_{3 j}, \mathbf{Y}_{4 i} \succeq 0, \mathbf{Y}_{4 i} \succeq \mathbf{Q}_{4 i}, \mathbf{Y}_{5 i} \succeq 0, \mathbf{Y}_{5 i} \succeq \mathbf{Q}_{5 i}$, respectively, the PLMFs method can be used for relaxing $\mathbf{Q}_{2 j}$ -
$\mathbf{Q}_{5 i}$. For the sake of simplicity, we will not show the derivation of these relaxed conditions. In terms of above analysis, the relaxed results are summarized in the following theorem.

Theorem 1: Given a positive T-S fuzzy model with time delay (2) and satisfying Lemma 1, a positive T-S fuzzy filter (4) exists such that the stability and positivity of the positive T-S fuzzy filter error system (5) can be ensured with satisfying performance index (6), if there exist slack matrices $\mathbf{Y}_{1 i j} \succeq 0$, $\mathbf{Y}_{2 j} \succeq 0, \mathbf{Y}_{3 j} \succeq 0, \mathbf{Y}_{4 i} \succeq 0, \mathbf{Y}_{5 i} \succeq 0$ as well as vectors $\mathbf{p}_{11} \succeq 0, \mathbf{p}_{12} \succeq 0, \lambda \succeq 0, \mathbf{p}_{2}$ and filter gain matrices $\mathbf{P}_{B f, j} \succeq$ $0, \mathbf{C}_{f j} \succeq 0 \mathbf{D}_{f j} \succeq 0$ and Metzler matrix $\mathbf{P}_{A f, j}$ satisfying:

$F_{1 i j, r k}$ is SOS, $\forall i, j, r, k$;

$F_{2 i j, r k} \quad$ is SOS, $\forall i, j, r, k$;

$F_{3 i j, r k}$ is $\operatorname{SOS}, \forall i, j, r, k$;

$F_{4 i j, r k}$ is SOS, $\forall i, j, r, k$;

$F_{5 i j, r k}$ is SOS, $\forall i, j, r, k$;

$F_{6 i j, r k}$ is $\operatorname{SOS}, \forall i, j, r, k$;

$\rho^{T}\left(\operatorname{diag}\left(\mathbf{Y}_{1 i j}-\mathbf{Q}_{1 i j}\right)\right) \rho$ is SOS, $\forall i, j ;$

$\rho^{T}\left(\operatorname{diag}\left(\mathbf{Y}_{2 j}-\mathbf{Q}_{2 j}\right)\right) \rho$ is $\operatorname{SOS}, \forall j$;

$\sigma^{T}\left(\operatorname{diag}\left(\mathbf{Y}_{3 j}-\mathbf{Q}_{3 j}\right)\right) \sigma \quad$ is $\operatorname{SOS}, \forall j$;

$v^{T}\left(\operatorname{diag}\left(\mathbf{Y}_{4 i}-\mathbf{Q}_{4 i}\right)\right) v \quad$ is $\mathbf{S O S}, \forall i$;

$\rho^{T}\left(\operatorname{diag}\left(\mathbf{Y}_{5 i}-\mathbf{Q}_{5 i}\right)\right) \rho$ is $\mathbf{S O S}, \forall i$;

$-\rho^{T}\left(\operatorname{diag}\left(\sum_{i=1}^{p} \sum_{j=1}^{c}\left(\left(\mu_{i}\left(D_{i_{1} i_{2} \cdots i_{2 n} s}\right) \eta_{j}\left(D_{i_{1} i_{2} \cdots i_{2 n} s}\right)+\gamma_{i j s}\right) \mathbf{Q}_{1 i j}\right.\right.\right.$

$\left.\left.\left.+\left(\beta_{i j s}-\gamma_{i j s}\right) \mathbf{Y}_{1 i j}+\epsilon_{1} \mathbf{I}_{n}\right)\right)\right) \rho \quad$ is $\operatorname{SOS}, \forall i, j$

$-\rho^{T}\left(\operatorname{diag}\left(\sum_{i=1}^{p} \sum_{j=1}^{c}\left(\left(\mu_{i}\left(D_{i_{1} i_{2} \cdots i_{2 n} s}\right) \eta_{j}\left(D_{i_{1} i_{2} \cdots i_{2 n} s}\right)+\gamma_{i j s}\right) \mathbf{Q}_{2 j}\right.\right.\right.$

$\left.\left.\left.+\left(\beta_{i j s}-\gamma_{i j s}\right) \mathbf{Y}_{2 j}+\epsilon_{2} \mathbf{I}_{n}\right)\right)\right) \rho \quad$ is $\operatorname{SOS}, \forall j$;

$-\sigma^{T}\left(\operatorname{diag}\left(\sum_{i=1}^{p} \sum_{j=1}^{c}\left(\left(\mu_{i}\left(D_{i_{1} i_{2} \cdots i_{2 n} s}\right) \eta_{j}\left(D_{i_{1} i_{2} \cdots i_{2 n} s}\right)+\gamma_{i j s}\right) \mathbf{Q}_{3 j}\right.\right.\right.$

$\left.\left.\left.+\left(\beta_{i j s}-\gamma_{i j s}\right) \mathbf{Y}_{3 j}+\epsilon_{3} \mathbf{I}_{l}\right)\right)\right) \sigma \quad$ is $\operatorname{SOS}, \forall j$;

$-v^{T}\left(\operatorname{diag}\left(\sum_{i=1}^{p} \sum_{j=1}^{c}\left(\left(\mu_{i}\left(D_{i_{1} i_{2} \cdots i_{2 n} s}\right) \eta_{j}\left(D_{i_{1} i_{2} \cdots i_{2 n} s}\right)+\gamma_{i j s}\right) \mathbf{Q}_{4 i}\right.\right.\right.$

$\left.\left.\left.+\left(\beta_{i j s}-\gamma_{i j s}\right) \mathbf{Y}_{4 i}+\epsilon_{4} \mathbf{I}_{m}\right)\right)\right) v \quad$ is $\operatorname{SOS}, \forall i$;

$-\rho^{T}\left(\operatorname{diag}\left(\sum_{i=1}^{p} \sum_{j=1}^{c}\left(\left(\mu_{i}\left(D_{i_{1} i_{2} \cdots i_{2 n} s}\right) \eta_{j}\left(D_{i_{1} i_{2} \cdots i_{2 n} s}\right)+\gamma_{i j s}\right) \mathbf{Q}_{5 i}\right.\right.\right.$

$\left.\left.\left.+\left(\beta_{i j s}-\gamma_{i j s}\right) \mathbf{Y}_{5 i}+\epsilon_{5} \mathbf{I}_{n}\right)\right)\right) \rho \quad$ is SOS, $\forall i$;

where $\mathbf{Y}_{1 i j}, \mathbf{Y}_{2 j}, \mathbf{Y}_{3 j}, \mathbf{Y}_{4 i}, \mathbf{Y}_{5 i} ; \mathbf{p}_{11}, \mathbf{p}_{12}, \mathbf{p}_{2}, \lambda ; \mathbf{P}_{B f, j}$, 
$\mathbf{C}_{f j}, \mathbf{D}_{f j}$ and $\mathbf{P}_{A f, j}$ are to be determined. $\rho \in \Re^{n}, \sigma \in$ $\Re^{l}$ and $v \in \Re^{m}$ are arbitrary vectors, $\epsilon_{1}, \epsilon_{2}, \epsilon_{3}, \epsilon_{4}$ and $\epsilon_{5}$ are predefined positive scalars. $\mathbf{I}_{n} \in \Re^{n}, \mathbf{I}_{l} \in \Re^{l}$ and $\mathbf{I}_{m} \in$ $\Re^{m}$ are vectors with all the elements being $1 . F_{1 i j, r k}, F_{2 i j, r k}$, $F_{3 i j, r k}, F_{4 i j, r k}, F_{5 i j, r k}$ and $F_{6 i j, r k}$ are the $r$-th row and $k$ th column element in $\mathbf{F}_{1 i j}, \mathbf{F}_{2 i j}, \mathbf{F}_{3 i j}, \mathbf{F}_{4 i j}, \mathbf{F}_{5 i j}$ and $\mathbf{F}_{6 i j}$, respectively. $\mathbf{Q}_{1 i j}, \mathbf{Q}_{2 j}, \mathbf{Q}_{3 j}, \mathbf{Q}_{4 i}$ and $\mathbf{Q}_{5 i}$ can be found in (52)-(56). $\mathbf{A}_{f j}$ and $\mathbf{B}_{f j}$ can be calculated based on (30).

\section{Simulation Example}

In this section, an example is given to demonstrate the effectiveness of the designed positive fuzzy filter. As described in Assumption 3, in the following, a positive T-S fuzzy model is given in the form of (2) with three rules:

$$
\begin{aligned}
& \mathbf{A}_{1}=\left[\begin{array}{cc}
-2.84 & 1.42 \\
0.26 & -1.3
\end{array}\right], \mathbf{A}_{2}=\left[\begin{array}{cc}
-1.01 & 1.55 \\
0.38 & -1.95
\end{array}\right], \\
& \mathbf{A}_{3}=\left[\begin{array}{cc}
-1.51 & 0.36 \\
0.16 & -1.48
\end{array}\right], \mathbf{A}_{d 1}=\left[\begin{array}{ll}
0.01 & 0.00 \\
0.01 & 0.08
\end{array}\right], \\
& \mathbf{A}_{d 2}=\left[\begin{array}{cc}
0.01 & 0.00 \\
0.02 & 0.06
\end{array}\right], \mathbf{A}_{d 3}=\left[\begin{array}{ll}
0.01 & 0.00 \\
0.03 & 0.07
\end{array}\right], \\
& \mathbf{A}_{\omega 1}=\left[\begin{array}{c}
0.01 \\
0.1
\end{array}\right], \mathbf{A}_{\omega 2}=\left[\begin{array}{l}
0.01 \\
0.09
\end{array}\right], \mathbf{A}_{\omega 3}=\left[\begin{array}{l}
0.02 \\
0.05
\end{array}\right], \\
& \mathbf{x}=\left[\begin{array}{ll}
x_{1} & x_{2}
\end{array}\right]^{T}, \mathbf{C}_{1}=\left[\begin{array}{ll}
1.01 & 1.01
\end{array}\right], \\
& \mathbf{C}_{2}=\left[\begin{array}{ll}
4.45 & 4.83
\end{array}\right], \mathbf{C}_{3}=\left[\begin{array}{ll}
5.56 & 5.82
\end{array}\right], \\
& \mathbf{C}_{\omega 1}=0.01, \mathbf{C}_{\omega 2}=0.02, \mathbf{C}_{\omega 3}=0.03, \mathbf{C}_{d 1}=\left[\begin{array}{ll}
0.01 & 0.01
\end{array}\right], \\
& \mathbf{C}_{d 2}=\left[\begin{array}{ll}
0.01 & 0.02
\end{array}\right], \mathbf{C}_{d 3}=\left[\begin{array}{ll}
0.01 & 0.03
\end{array}\right], \\
& \mathbf{E}_{1}=\left[\begin{array}{ll}
5.93 & 6.33
\end{array}\right], \mathbf{E}_{2}=\left[\begin{array}{ll}
1.87 & 3.41
\end{array}\right], \\
& \mathbf{E}_{3}=\left[\begin{array}{ll}
2.69 & 1.55
\end{array}\right], \\
& \mathbf{E}_{\omega 1}=0.01, \mathbf{E}_{\omega 2}=0.02, \mathbf{E}_{\omega 3}=0.02, \mathbf{E}_{d 1}=\left[\begin{array}{ll}
0.01 & 0.01
\end{array}\right], \\
& \mathbf{E}_{d 2}=\left[\begin{array}{ll}
0.02 & 0.01
\end{array}\right], \mathbf{E}_{d 3}=\left[\begin{array}{ll}
0.02 & 0.01
\end{array}\right],
\end{aligned}
$$

As previously mentioned, $\mathbf{F}_{d}$ is a given matrix whose elements satisfy $F_{d, r k}=\max \left\{a_{d i, r k}\right\}$, where $a_{d i, r k}$ is the $r$ th row and $k$-th column element of $\mathbf{A}_{d i}$ and $F_{d, r k}$ is the $r$-th row and $k$-th column element of $\mathbf{F}_{d}$. The disturbance is chosen as $\tilde{\mathbf{w}}(t)=\beta e^{-t}|\cos (2 t)|$, where $\beta$ is a constant scalar which is given reasonably. The MFs of the positive T-S fuzzy model with time delay are chosen as $w_{1}\left(x_{1}\right)=1-\frac{1}{1+e^{-\left(x_{1}-9\right) / 3}}$, $w_{3}\left(x_{1}\right)=\frac{1}{1+e^{-\left(x_{1}-1\right) / 3}}, w_{2}\left(x_{1}\right)=1-w_{1}\left(x_{1}\right)-w_{3}\left(x_{1}\right)$. And the MFs of positive T-S fuzzy filter are chosen as $m_{1}\left(x_{f 1}\right)=$ $1-\frac{1}{1+0.5 e^{-\left(x_{f 1}-10\right)}}, m_{2}\left(x_{f 1}\right)=1-m_{1}\left(x_{f 1}\right)$. When the PLMFs method is considered, we pick out the sample points of $w_{i}\left(x_{1}\right)$ at $x_{1} \in\{0,4,8,12,16,20\}, i=1,2,3$ and the sampled points of $m_{j}\left(x_{f 1}\right)$ at $x_{f 1} \in\{0,4,8,12,16,20\}$, $j=1,2$, which means the operating domain of positive T$\mathrm{S}$ fuzzy model and the one of positive T-S fuzzy filter are divided into 5 sub-domains, respectively.

In terms of the Theorem 1, the optimal performance index is calculated as $\gamma=3.4997$ and the filter gain matrices are given in the following. Furthermore, the time responses of $x_{1}, x_{f 1}$, $x_{2}, x_{f 2}, z, z_{f}$ and $\tilde{z}$ are acquired and shown in Figs. 1 to 8 . In these figures, different time responses of estimation errors
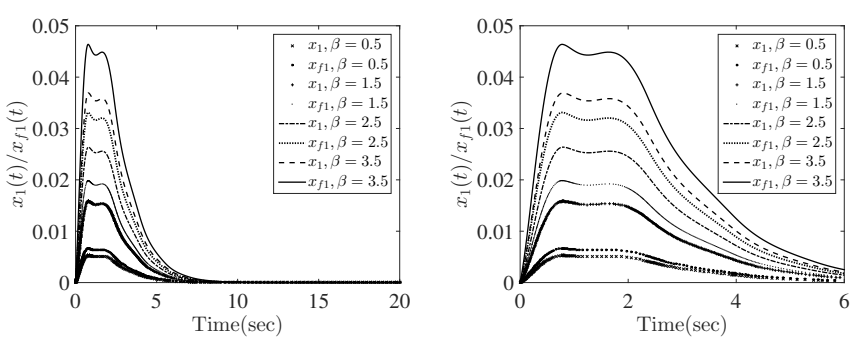

Fig. 1. System state $x_{1}(t)$ and filter Fig. 2. Drawing of partial enlargestate $x_{f 1}(t)$ for different $\beta$ ment of system state $x_{1}(t)$ and filter state $x_{f 1}(t)$ for different $\beta$
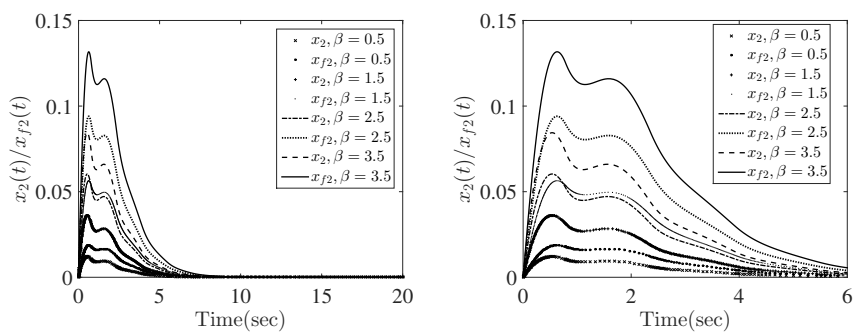

Fig. 3. System state $x_{2}(t)$ and filter state $x_{f 2}(t)$ for different $\beta$

Fig. 4. Drawing of partial enlargement of system state $x_{2}(t)$ and filter state $x_{f 2}(t)$ for different $\beta$

and state variables are displayed when the constant coefficient $\beta$ in the disturbance are different.

$$
\begin{aligned}
& \mathbf{A}_{f 1}=\left[\begin{array}{cc}
-4.2012 & 3.6304 \times 10^{-2} \\
2.6813 \times 10^{-4} & -11.4001
\end{array}\right], \\
& \mathbf{A}_{f 2}=\left[\begin{array}{cc}
-4.2095 & 3.2038 \times 10^{-2} \\
2.2390 \times 10^{-4} & -11.4001
\end{array}\right], \\
& \mathbf{B}_{f 1}=\left[\begin{array}{cc}
1.4010 \\
10.0002
\end{array}\right], \mathbf{B}_{f 2}=\left[\begin{array}{c}
1.4002 \\
10.0002
\end{array}\right], \\
& \mathbf{C}_{f 1}=\left[\begin{array}{cc}
1.6538 \times 10^{-5} & 4.0000 \times 10^{-1}
\end{array}\right], \\
& \mathbf{C}_{f 2}=\left[\begin{array}{ll}
1.2540 \times 10^{-5} & 3.9999 \times 10^{-1}
\end{array}\right], \\
& \mathbf{D}_{f 1}=5.8713, \mathbf{D}_{f 2}=5.8713 .
\end{aligned}
$$

From the time response of the systems states and filter states, we can see that the positive T-S fuzzy filter can make the time response of the positive T-S fuzzy filter system with time delay quickly close to zero, which means the positive T-S fuzzy filter can achieve asymptotic stability and positivity of the positive T-S fuzzy filter error system. In addition, from Figs. 1 to 8 , it can be seen that with the value of $\beta$ becoming smaller, the time responses of $x_{f 1}, x_{f 2}, z_{f}$ are closer to $x_{1}, x_{2}$, $z$, respectively. Hence, we can draw a conclusion that when the value of $\beta$ is smaller, the filter effectiveness is better. Besides, when the disturbance parameter $\beta$ gets bigger, the convergence rates of the filter state variables and system state variables slow down. And the estimation errors will be bigger with the disturbance becoming stronger. Therefore, it can be concluded that the smaller the disturbance parameter $\beta$, the better the convergence effect of the positive T-S fuzzy filter error system. 

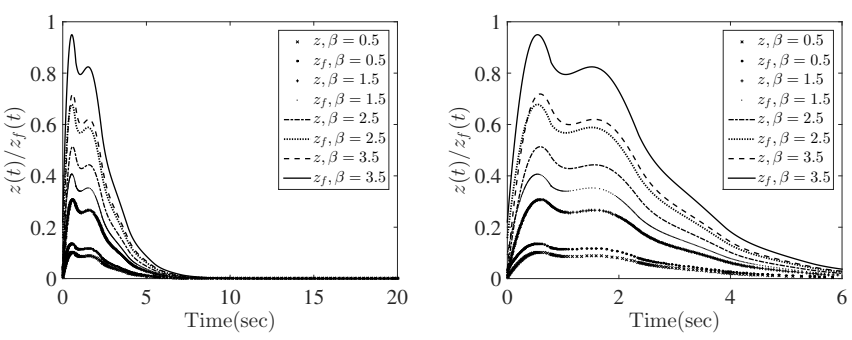

Fig. 5. Output $z(t)$ and the estimated Fig. 6. Drawing of partial enlargeoutput $z_{f}(t)$ for different $\beta$ ment of output $z(t)$ and the estimated output $z_{f}(t)$ for different $\beta$
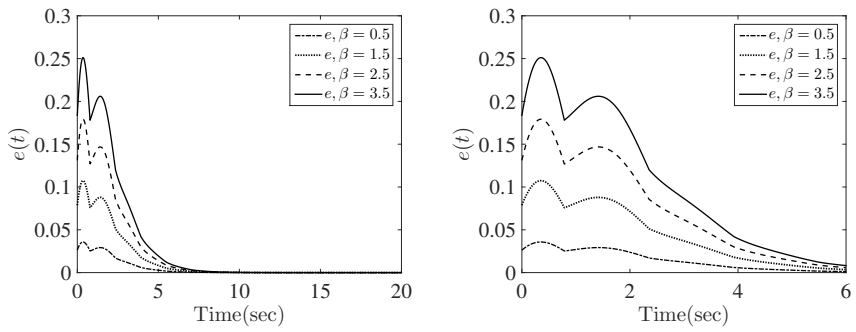

Fig. 7. Estimated errors $e(t)$ for dif- Fig. 8. Drawing of partial enlargeferent $\beta$ ment of estimated errors $e(t)$ for different $\beta$

\section{CONCLUSION}

In this paper, a positive T-S fuzzy filter has been designed for the positive T-S fuzzy system with time delay so that the stability and positivity analysis of the positive T-S fuzzy filter error system can be ensured under $L_{1}$-induced performance. By employing the augmented approach and the linear copositive Lyapunov theory, the stability and positivity conditions with satisfying the optimal performance index $\gamma$ have been obtained. Furthermore, considering the conservativeness, the advanced PLMFs method has been taken into account, which has a great help to explore some useful information inlaid in the MFs and facilitate the stability and positivity analysis. Ultimately, a simulation has been given to verify the validity of the strategy in this paper.

\section{REFERENCES}

[1] M. A. Rami and F. Tadeo, "Controller synthesis for positive linear systems with bounded controls," IEEE Trans. Circuits Syst., vol. 54, no. 2, pp. 151-155, Feb. 2007.

[2] Y. Ju, X. Zhu, and Y. Sun, "Stability analysis of continuous-time positive switched linear systems," in Proc. 18th Inter. Confer. Control, Auto. and Syst., Oct. 2018, pp. 1062-1065.

[3] V. T. Huynh, A. Arogbonlo, H. Trinh, and A. M. T. Oo, "Design of observers for positive systems with delayed input and output information," IEEE Trans. Circuits and Syst. II: Express Briefs, vol. 67, no. 1, pp. 107-111, Jan. 2020.

[4] M. E. Valcher, "Controllability and reachability criteria for discrete time positive systems," International Journal of Control, vol. 65, no. 3, pp. 511-536, 1996.

[5] P. G. Coxson and H. Shapiro, "Positive input reachability and controllability of positive systems," Linear Algebra and its Appli., vol. 94, pp. $35-53,1987$.

[6] L. Caccetta and V. G. Rumchev, "A survey of reachability and controllability for positive linear systems," Annals of Operations Research, vol. 98, pp. 101-122, Dec. 2000.
[7] B. D. O. Anderson, M. Deistler, L. Farina, and L. Benvenuti, "Nonnegative realization of a linear system with nonnegative impulse response," IEEE Trans. Circuits and Syst. I: Fundamental Theory and Appli., vol. 43, no. 2, pp. 134-142, Feb. 1996.

[8] L. Benvenuti and L. Farina, "A note on minimality of positive realizations," IEEE Trans. Circuits and Syst. I: Fundamental Theory and Appli., vol. 45, no. 6, pp. 676-677, Jun. 1998

[9] M. Kaykobad, "Positive solutions of positive linear systems," Linear Algebra and its Appli., vol. 64, pp. 133 - 140, 1985.

[10] J. Shen and J. Lam, "Static output-feedback stabilization with optimal $l_{1}$-gain for positive linear systems," Automatica, vol. 63, pp. $248-253$, 2016.

[11] R. H. Middleton, P. Colaneri, E. Hernandez-Vargas, and F. Blanchini, "Continuous-time optimal control for switched positive systems with application to mitigating viral escape," IFAC Sympo. Nonlinear Control Syst., vol. 43, no. 14, pp. $266-271,2010$.

[12] P. Colaneri, R. H. Middleton, Z. Chen, D. Caporale, and F. Blanchini, "Convexity of the cost functional in an optimal control problem for a class of positive switched systems," Automatica, vol. 50, no. 4, pp. 1227 - 1234, 2014.

[13] X. Liu, W. Yu, and L. Wang, "Stability analysis for continuous-time positive systems with time-varying delays," IEEE Trans. Autom. Control, vol. 55, no. 4, pp. 1024-1028, Apr. 2010.

[14] I. Zaidi, M. Chaabane, F. Tadeo, and A. Benzaouia, "Static statefeedback controller and observer design for interval positive systems with time delay," IEEE Trans. Circuits and Syst. II: Express Briefs, vol. 62, no. 5, pp. 506-510, May 2015.

[15] S. Bhattacharyya and S. Patra, "Static output-feedback stabilization for MIMO LTI positive systems using LMI-based iterative algorithms," IEEE Control Syst. Letters, vol. 2, no. 2, pp. 242-247, Apr. 2018.

[16] C. Y. Kao, "On output feedback control of positive linear systems," in Proc. the SICE Annual Confer., Sep. 2014, pp. 881-885.

[17] X. Zhao, T. Wu, X. Zheng, and R. Li, "Discussions on observer design of nonlinear positive systems via T-S fuzzy modeling," Neurocomputing, vol. 157, pp. $70-75,2015$.

[18] X. Chen, J. Lam, and P. Li, "Positive filtering for continuous-time positive systems under $l_{1}$ performance," Inter. Journal of Control, vol. 87, no. 9, pp. 1906-1913, Nov. 2014.

[19] S. Xiao, Y. Zhang, Q. Xu, and B. Zhang, "Event-triggered network-based $\ell_{1}$-gain filtering for positive linear systems," Inter. Journal of Systems Science, vol. 48, no. 6, pp. 1281-1290, 2017.

[20] C. Tseng, "Robust fuzzy filter design for a class of nonlinear stochastic systems," IEEE Trans. Fuzzy Syst., vol. 15, no. 2, pp. 261-274, Apr. 2007.

[21] J. An, G. Wen, C. Lin, and R. Li, "New results on a delay-derivativedependent fuzzy $H_{\infty}$ filter design for T-S fuzzy systems," IEEE Trans. Fuzzy Syst., vol. 19, no. 4, pp. 770-779, Aug. 2011.

[22] S. Huang, X. He, and N. Zhang, "New results on $H_{\infty}$ filter design for nonlinear systems with time delay via T-S fuzzy models," IEEE Trans. Fuzzy Syst., vol. 19, no. 1, pp. 193-199, Feb. 2011.

[23] M. Wang, J. Qiu, and G. Feng, "A novel piecewise affine filtering design for T-S fuzzy affine systems using past output measurements," IEEE Trans. Cybernetics, pp. 1-10, Dec. 2018.

[24] X. Chen, J. Lam, P. Li, and Z. Shu, "Output-feedback control for continuous-time interval positive systems under $L_{1}$ performance," $A p$ plied Mathe. and Computation., vol. 16, no. 6, pp. 1592-1601, Oct. 2014.

[25] W. Qi, X. Gao, and Y. Kao, "Positive $L_{1}$-gain filter design for positive markovian jump systems with time-varying delay and incomplete transition rates," Canadian Journal of Physics, vol. 94, no. 9, pp. 877-883, Jun. 2016.

[26] G. Chen, Q. Xie, and L. S. Shieh, "Fuzzy kalman filtering," Information Sciences, vol. 109, no. 1, pp. 197 - 209, 1998.

[27] D. Simon, "Kalman filtering for fuzzy discrete time dynamic systems," Applied Soft Computing, vol. 3, no. 3, pp. 191 - 207, 2003.

[28] J. An, G. Wen, and W. Xu, "Improved results on fuzzy $H_{\infty}$ filter design for T-S fuzzy systems," Discrete Dynamics in Nature and Society, vol. 2010, Aug. 2010.

[29] Y. Zheng, S. Sam Ge, and Y. Wang, "Non-weighted $l_{1}$ filtering for positive switched delay systems," IET Control Theory Appli., vol. 12, no. 8, pp. 1046-1054, 2018.

[30] W. M. Haddad, V. Chellaboina, and E. August, "Stability and dissipativity theory for nonnegative dynamical systems: a thermodynamic framework for biological and physiological systems," in Prof. 40th IEEE Confer. Decision and Control, vol. 1, Dec. 2001, pp. 442-458. 
[31] A. Benzaouia, F. Mesquine, M. Benhayoun, H. Schulte, and S. Georg, "Stabilization of positive constrained T-S fuzzy systems: Application to a buck converter," Journal of the Franklin Institute, vol. 351, no. 8, pp. $4111-4123,2014$.

[32] L. Imsland, B. A. Foss, and G. O. Eikrem, "State feedback control of a class of positive systems: Application to gas-lift stabilization," in 2003 European Control Conference (ECC), Sep. 2003, pp. 2499-2504.

[33] Y. Li and H. Zhang, "Asynchronous $L_{1}$-gain control of uncertain switched positive linear systems with dwell time," Isa Trans., vol. 75, pp. $25-37,2018$.

[34] A. Meng, H. K. Lam, Y. Yu, X. Li, and F. Liu, "Static output feedback stabilization of positive polynomial fuzzy systems," IEEE Trans. Fuzzy Syst., vol. 26, no. 3, pp. 1600-1612, Jun. 2018.

[35] A. Meng, H. Lam, F. Liu, C. Zhang, and P. Qi, "Output feedback and stability analysis of positive polynomial fuzzy systems," IEEE Trans. Syst., Man, and Cyber: Systems, pp. 1-12, 2020.

[36] A. Meng, H. K. Lam, L. Hu, and F. Liu, " $l_{1}$-induced static output feedback controller design and stability analysis for positive polynomial fuzzy systems," in Proc. the 19th UK Workshop on Computational Intelligence, vol. 1043, Sep. 2019, pp. 41-52.

[37] A. Chatterjee, R. Chatterjee, F. Matsuno, and T. Endo, "Augmented stable fuzzy control for flexible robotic arm using lmi approach and neuro-fuzzy state space modeling," IEEE Trans. Industrial Electronics, vol. 55, no. 3, pp. 1256-1270, 2008

[38] R. E. Precup, M. L. Tomescu, and C. A. Dragos, "Stabilization of rössler chaotic dynamical system using fuzzy logic control algorithm," Inter. Journal of General Syst., vol. 43, no. 5, pp. 413-433, 2014

[39] M. Radgolchin and H. Moeenfard, "Development of a multi-level adaptive fuzzy controller for beyond pull-in stabilization of electrostatically actuated microplates," Journal of Vibra. and Control, vol. 24, no. 5, pp. 860-878, Jun 2016.

[40] R. P. A. Gil, Z. C. Johanyák, and T. Kovács, "Surrogate model based optimization of traffic lights cycles and green period ratios using microscopic simulation and fuzzy rule interpolation," Inter. journal of artif. intelligence, vol. 16, pp. 20-40, 2018.

[41] T. Zhao, J. Xiao, H. Sheng, and W. Tao, " $H_{\infty}$ control of continuoustime interval type-2 T-S fuzzy systems via dynamic output feedback controllers," Neurocomputing, vol. 165, pp. 133-143, Oct. 2015.

[42] S. Du, J. Qiao, X. Zhao, and R. Wang, "Stability and $l_{1}$-gain analysis for switched positive T-S fuzzy systems under asynchronous switching," Journal of the Franklin Institute, vol. 355, no. 13, pp. 5912 - 5927, 2018

[43] S. Du and J. Qiao, "Stability analysis and $L_{1}$-gain controller synthesis of switched positive T-S fuzzy systems with time-varying delays," Neurocomputing, vol. 275, pp. 2616-2623, 2018.

[44] L. Wang and H. K. Lam, "New stability criterion for continuous-time Takagi-Sugeno fuzzy systems with time-varying delay," IEEE Trans. Cyber, vol. 49, no. 4, pp. 1551-1556, Apr. 2019.

[45] R. E. Precup, S. Doboli , and S. Preitl , "Stability analysis and development of a class of fuzzy control systems," Engineer. Appli. of Artif. Intelligence, vol. 13, no. 3, pp. 237 - 247, 2000.

[46] X. Chen, J. Lam, and H. K. Lam, "Positive filtering for positive Takagi-Sugeno fuzzy systems under $\ell_{1}$ performance," Information Sciences, vol. 299, pp. $32-41,2015$.

[47] Y. Chen, Y. Bo, and B. Du, "Positive $L_{1}$-filter design for continuoustime positive markov jump linear systems: full-order and reduced-order," IET Control Theory Appli., vol. 13, no. 12, pp. 1855-1862, 2019.

[48] R. Oubah and A. Benzaouia, "Stability and stabilization of positive Takagi-Sugeno fuzzy continuous systems with delay by linear programming," in proc. 5th Inter. Confer. Systems and Control, May 2016, pp. 90-96.

[49] E. Fornasini and M. E. Valcher, "Linear copositive lyapunov functions for continuous-time positive switched systems," IEEE Trans. Autom. Control, vol. 55, no. 8, pp. 1933-1937, Aug. 2010.

[50] X. Liu, "Stability analysis of switched positive systems: A switched linear copositive lyapunov function method," IEEE Trans. Circuits and Systems II: Express Briefs, vol. 56, no. 5, pp. 414-418, May 2009.

[51] L. H. K. and M. Narimani, "Quadratic-stability analysis of fuzzy-modelbased control systems using staircase membership functions," IEEE Trans. Fuzzy Syst., vol. 18, no. 1, pp. 125-137, Feb. 2010.

[52] M. Narimani and H. K. Lam, "SOS-based stability analysis of polynomial fuzzy-model-based control systems via polynomial membership functions," IEEE Trans. Fuzzy Syst., vol. 18, no. 5, pp. 862-871, Oct. 2010.

[53] H. K. Lam, "Polynomial fuzzy-model-based control systems: Stability analysis via piecewise-linear membership functions," IEEE Trans. Fuzzy Syst., vol. 19, no. 3, pp. 588-593, Jun. 2011.
[54] H. K. Lam, "A review on stability analysis of continuous-time fuzzymodel-based control systems: From membership-function-independent to membership-function-dependent analysis," Engineering Appli. of Artif. Intelligence, vol. 67, pp. 390 - 408, Jan. 2018.

[55] P. Li, J. Lam, and Z. Shu, " $H_{\infty}$ positive filtering for positive linear discrete-time systems: An augmentation approach," IEEE Trans. Autom. Control, vol. 55, no. 10, pp. 2337-2342, Oct. 2010.

[56] L. Farina and S. Rinaldi, Positive linear systems: Theory and appli. New York: John Wiley and Sons Inc., 2000.

[57] J. Zhang, Z. Han, F. Zhu, and J. Huang, "Brief paper: feedback control for switched positive linear systems," IET Control Theory and Appli. vol. 7, no. 3, pp. 464-469, Jun. 2013.

[58] X. Chen, Analysis and Synthesis of Positive Systems Under $\ell_{1}$ and $L_{1}$ Performance. Springer Publishing Company, 2016.

[59] Y. Zhao, B. Xiao, C. Liu, H. Li, and H. K. Lam, "Relaxed LMI-based stability conditions for fuzzy-model-based control systems under imperfect premise matching: Approximated membership function approach," in Proc. the 11th World Congress on Intelligent Control and Automation, Jun. 2014, pp. 251-256.

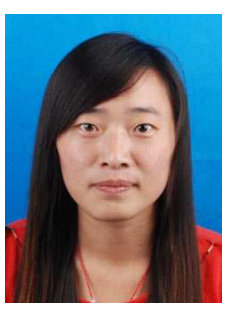

Aiwen Meng received the B.Eng. degree in automation from Liren College, Yanshan University, Hebei, China, in 2015. She is currently pursuing the Ph.D. degree in control science and engineering from Yanshan University, Hebei, China and on an academic exchange at De Montfort University, Leicester, U.K. as an honorary research assistant. The field of study for the Ph.D. degree is the control synthesis of positive nonlinear systems.

Her research interests include intelligent control systems and networked control systems.

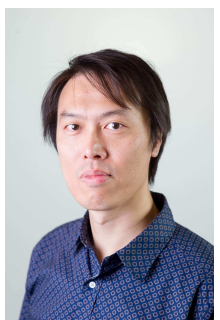

H. K. Lam (M'98-SM'12-F'20) received the B.Eng. (Hons.) and Ph.D. degrees from the Department of Electronic and Information Engineering, The Hong Kong Polytechnic University, Hong Kong, in 1995 and 2000, respectively. During the period of 2000 and 2005, he worked with the Department of Electronic and Information Engineering at The Hong Kong Polytechnic University as Post-Doctoral Fellow and Research Fellow respectively. He joined as a Lecturer at King's College London in 2005 and is currently a Reader

His current research interests include intelligent control systems and computational intelligence. He has served as a program committee member and international advisory board member for various international conferences and a reviewer for various books, international journals and international conferences. He is an associate editor for IEEE Transactions on Fuzzy Systems, IEEE Transactions on Circuits and Systems II: Express Briefs, IET Control Theory and Applications, International Journal of Fuzzy Systems and Neurocomputing; and guest editor for a number of international journals, and is in the editorial board of for a number of international journals. He is an IEEE Fellow.

$\mathrm{He}$ is the coeditor for two edited volumes: Control of Chaotic Nonlinear Circuits (World Scientific, 2009) and Computational Intelligence and Its Applications (World Scientific, 2012), and the coauthor of the monographs: Stability Analysis of Fuzzy-Model-Based Control Systems (Springer, 2011), Polynomial Fuzzy Model Based Control Systems (Springer, 2016) and Analysis and Synthesis for Interval Type-2 Fuzzy-Model-Based Systems (Springer, 2016). 


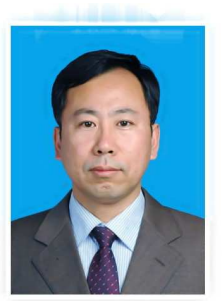

Fucai Liu received the B.S. and M.S. degrees from both Department of Automation, Northeast Heavy Mechanism Academe, Qiqihar, China, in 1989 and 1994. He received the Ph.D. degree from Department of control science and Engineering, Harbin Institute of Technology in 2003. He is now a professor of Yanshan and the head of Automation Department in Electric Engineering Institute at Yanshan University. He has authored/co-authored more than 200 papers in mathematical, technical journals, and conferences. $\mathrm{He}$ is the author of the book "Fuzzy Model Identification for Nonlinear Systems and Its Applications". His current research interests include fuzzy identification, predict control and space robot Control.

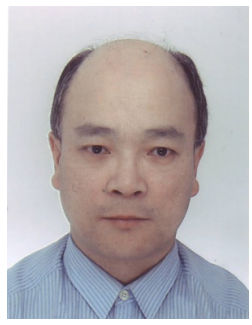

Yingjie Yang received the B.Sc., M.Sc. and Ph.D. degrees in engineering from Northeastern University (China) in 1987, 1990, and 1994, respectively. He was awarded his $\mathrm{PhD}$ degree in computer science at Loughborough University (UK) in 2008 . He is currently a Professor of Computational Intelligence at the Institute of Artificial Intelligence of De Montfort University. He has published over 160 papers on grey systems, fuzzy sets, rough sets, neural networks and their applications to civil engineering, transportation and environmental engineering. His research interests include the representation and modeling of various uncertainties, and the application of computational intelligence to real world problems.

$\mathrm{He}$ is the executive president of the International Association of Grey Systems and Uncertainty Analysis, a senior member of IEEE Systems, Man and Cybernetics Society, a co-chair of IEEE SMC Technical Committee on Grey Systems. He has chaired and co-chaired a number of international conferences and special sessions, and been involved as programme committee member in over 100 international conferences. He is serving as an associate editor in several international academic journals including IEEE Transaction on Cybernetics. 